\title{
HYPOELLIPTICITY ON CAUCHY-RIEMANN MANIFOLDS
}

\author{
JOHANNES A. PETERSEN
}

\begin{abstract}
Using a recent homotopy formula by Trèves, it is shown that the existence of $(q+1)$-dimensional holomorphic supporting manifolds is a sufficient condition for the hypoellipticity on level $q$ and $n-q$ of a tangential Cauchy-Riemann complex of CR-dimension $n$. In the hypersurface case, this result is given microlocally.
\end{abstract}

\section{INTRODUCTION}

It is known that a CR-structure on a manifold $\mathscr{M}$ is $q$-hypoelliptic (see Definition 2.1), provided its Levi form is nondegenerate and has at least $q+$ 1 eigenvalues of each sign (see [H1, 2, He, FS, §17]). In the present work we show that the nondegeneracy of the Levi form is not essential and give a sufficient condition for hypoellipticity which can do without that assumption. The condition on the signs of the eigenvalues of the Levi form is replaced by its geometrical generalization, namely, the existence of holomorphic supporting manifolds tangent to the local embedding $\Sigma \subset \mathbf{C}^{m}$ of $\mathscr{M}$.

The main tool which we shall use to prove our theorems comes from [T3]. It is an adaptation of the Bochner-Martinelli-Koppelmann homotopy formulas to CR-structures. These formulas allow us to handle the tangential CauchyRiemann operator $\bar{\partial}_{\Sigma}$ roughly speaking as follows: we can write $u=K \bar{\partial}_{\Sigma} u \dot{+}$ $\bar{\partial}_{\Sigma} K u+$ error terms, and from there, under the assumption that $\bar{\partial}_{\Sigma} u$ is a smooth form, prove that $u$ is smooth (modulo coboundaries). Of course, to do this we shall need to assume certain properties of the kernel $K$, which in turn will depend on the geometry of $\Sigma$.

We remark that Nagel and Rosay (1989) have shown that unfortunately exact homotopy formulas do not exist in general.

For the convenience of the reader, we shall begin by restating carefully a homotopy formula with error terms for the operator $\bar{\partial}_{\Sigma}$, which has proven in [T3]. The main ingredient is a differential form $E_{a}$ related to the fundamental solution of the Laplacian, which we define in $\S 1.2$. The formula given here has two error terms, one of which vanishes under certain conditions (specified in $\S 1.3)$ and the other turns out to be benign.

We then turn to the question of hypoellipticity. The definition used here is the natural one, that is, we call $\bar{\partial}_{\Sigma} q$-hypoelliptic if for any current $v$ of bidegree $(0, q)$ the smoothness of $\bar{\partial}_{\Sigma} v$ implies that $v$ is cohomologous to a smooth

Received by the editors June 12, 1990.

1991 Mathematics Subject Classification. Primary 32C16, 32F20; Secondary 35N15.

Work supported in part by NSF Grant DMS-8603171. 
form. In $\S 2$, we investigate the case of codimension one and define a condition for the real hypersurface $\Sigma$ which guarantees the hypoellipticity of $\bar{\partial}_{\Sigma}$. The condition is not dependent on the nondegeneracy of the Levi form. It is slightly less general than the condition given in [T3, Definition III.2.2] but geometrically simpler. We prove that $\bar{\partial}_{\Sigma}$ is $q$-hypoelliptic if $\Sigma$ allows $(q+1)$-dimensional holomorphic supporting manifolds on both sides. Half of this hypothesis also yields half of the conclusion, namely the microlocal hypoellipticity of $\bar{\partial}_{\Sigma}$ in one part of the characteristic set. The proof of Theorem 2.7 relies on the homotopy formula as well as on a technical lemma, Lemma 2.9, bridging the gap between geometry and analysis, and another analytical result, Lemma 2.14. The latter has been proven in [T3], and we outline the proof here for completeness.

Finally, in the last section, we try to make things work without any restrictions on the codimension of $\Sigma$ in $\mathbf{C}^{m}$. We were not able to do this microlocally, because boundary terms pop up in the transformation of the integrals in Lemma 3.13 if we integrate only over subsets of $\mathbf{R}_{d}$. Our theorem therefore yields hypoellipticity only if, roughly speaking, $\Sigma$ has some convexity in all directions $\sigma \in S_{d-1}$. Theorem 3.4 also applies in the case of a degenerate Levi form and thanks to Proposition 3.3 it generalizes a result by Trèves (loc. cit., hypothesis III.4.1). In the proof we follow as closely as possible the arguments given before in the hypersurface case.

Notations. $\quad>, \leqslant \quad$ strict inequality, with an obvious exception (cf. (2.8), (3.4)),

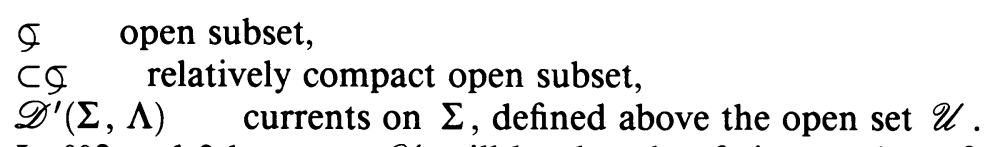

In $\S \S 2$ and 3 however, $\mathscr{U}$ will be shrunk a finite number of times and then elements of $\mathscr{D}^{\prime}(\Sigma, \Lambda)$ are meant to be germs at 0 .

$\mathscr{D}^{\prime}\left(\Sigma, \Lambda^{p, q}\right) \quad$ (germs of) currents of bidegree $(p, q)$ defined near 0 on $\Sigma$,

$\operatorname{Re} w, \operatorname{Im} w \quad$ real and imaginary part of $w$, $S^{-\infty}(\mathscr{U}) \quad$ symbols of regularizing operators (cf. [T1, p. 31]), $\langle\sigma\rangle:=\sqrt{\sum_{j=1}^{d} \sigma_{j}^{2}}$

$H(\sigma)$ Heaviside function, $T_{P}^{\mathrm{C}} \Sigma=\left\{t \in \mathbf{C}^{m}|\partial r|_{P}(t)=0\right\} \quad$ complex tangent space of $\Sigma=\{r=0\}$ at $P$.

\section{A HOMOTOPY FORMULA FOR CAUCHY-RIEMANN STRUCTURES}

1.1. Preliminaries. Let $1 \leq n \leq m$ and let $\mathscr{M}$ be a differentiable manifold of dimension $m+n$, carrying a CR-structure given by the functions

$$
\begin{array}{ll}
z: \mathscr{M} \rightarrow \mathbf{C}^{m} ; & (x, y, s) \mapsto x+i y, \\
w: \mathscr{M} \rightarrow \mathbf{C}^{d} ; & (x, y, s) \mapsto s+i \varphi(x, y, s) .
\end{array}
$$

We assume $d=m-n$, and $\varphi: \mathscr{M} \rightarrow \mathbf{R}^{d}$ is smooth with $\varphi(0)=0, d \varphi(0)=0$. The map $p:=(z, w): \mathscr{M} \rightarrow \mathbf{C}^{m}$ is called a fine local embedding of $\mathscr{M}$. Then the submanifold $\Sigma \stackrel{\text { def }}{=} p(\mathscr{M})$ of $\mathbf{C}^{m}$ is parametrized locally by $(z, s)$. We shall always assume $(z, s) \in \mathscr{U}$ an open neighbourhood of zero in $\mathbf{C}^{n} \times \mathbf{R}^{d}$, but 
henceforth we shall write $\mathscr{U}$ only when we want to emphasize the fact that the whole discussion is local; otherwise we shall write $\Sigma$.

The tangent structure bundle on $\mathscr{M}$ is generated by vector fields $L_{j}$ orthogonal to the forms $d z_{j}, d w_{k}$, for $1 \leq j \leq n, 1 \leq k \leq d$. Let us put

${ }^{t} \varphi_{\bar{z}_{j}}=\left(\frac{\partial \varphi_{1}}{\partial \bar{z}_{j}}, \ldots, \frac{\partial \varphi_{d}}{\partial \bar{z}_{j}}\right), \quad w_{\bar{z}}=i\left(\varphi_{\bar{z}_{1}}, \ldots, \varphi_{\bar{z}_{n}}\right), \quad \partial_{s}=\left(\frac{\partial}{\partial s_{1}}, \ldots, \frac{\partial}{\partial s_{d}}\right)$,

and

$$
w_{s}=\left(1+i \varphi_{s}\right)=\left(\delta_{k}+i \frac{\partial \varphi_{k l}}{\partial s_{l}}\right)_{1 \leq k, l \leq d} .
$$

Then we can choose the vector fields

$$
L_{j}=\frac{\partial}{\partial \bar{z}_{j}}-i^{t} \varphi_{\bar{z}_{j}}{ }^{t}\left(1+i \varphi_{s}\right)^{-1}{ }^{t} \partial_{s}, \quad \text { for } 1 \leq j \leq n
$$

The tangential Cauchy-Riemann operator is defined as

$$
\forall f \in \mathscr{D}^{\prime}(\Sigma, \Lambda) \quad \bar{\partial}_{\Sigma} f \stackrel{\text { def }}{=} \sum_{j=1}^{n} d \bar{z}_{j} \wedge L_{j} f .
$$

Let $0 \leq q \leq n$. We are interested in currents $f$ of bidegree $(m, q)$ on $\Sigma$. Remark 1.1. Each current $f \in \mathscr{D}^{\prime}\left(\Sigma, \Lambda^{m, q}\right)$ can be written in the form $f=$ $\sum_{|J|=q} f_{J}(z, s) d \bar{z}_{J} \wedge d z \wedge d w$. Its exterior derivative is then equal to

$$
d f=\sum_{|J|=q} \sum_{j=1}^{n} L_{j} f_{J} d \bar{z}_{j} \wedge d \bar{z}_{J} \wedge d z \wedge d w=\bar{\partial}_{\Sigma} f \in \mathscr{D}^{\prime}\left(\Sigma, \Lambda^{m, q+1}\right) .
$$

All commutators $\left[L_{i}, L_{j}\right]$ are zero.

We introduce the following notation. $J=\left(j_{1}, \ldots, j_{\nu+1}\right)$ shall denote an ordered subset of $\{1, \ldots, n\}$ of length $|J|=\nu+1 . J^{*}$ is its complement, naturally ordered.

Let $a: \mathscr{U}_{z, s} \times \mathscr{U}_{z^{\prime}, s^{\prime}} \times\left(\mathbf{R}_{d}\right)_{\sigma} \rightarrow \mathbf{C}_{n}$ be a smooth map. Let $d, d^{\prime}$ be the exterior derivatives on $\mathscr{U}_{z, s}, \mathscr{U}_{z^{\prime}, s^{\prime}}$ respectively, and $D:=d+d^{\prime}$ the exterior derivative on $\mathscr{U} \times \mathscr{U}$. We put $D a_{J}:=D a_{j_{1}} \wedge \cdots \wedge D a_{j_{\nu+1}}$, where

$$
\begin{aligned}
D a_{j}= & \sum_{i=1}^{n}\left(\frac{\partial a_{j}}{\partial z_{i}} d z_{i}+\cdots+\frac{\partial a_{j}}{\partial \bar{z}_{i}^{\prime}} d \bar{z}_{i}^{\prime}\right)+\frac{\partial a_{j}}{\partial s} d s+\frac{\partial a_{j}}{\partial s^{\prime}} d s \\
& \in C^{\infty}\left(\mathscr{U} \times \mathscr{U} \times \mathbf{R}_{d}, \Lambda^{1}\right) .
\end{aligned}
$$

It is the freedom of choice of this map $a$ that will make our proofs work later on.

Let $L_{j}^{\prime}$ be the analogue of $L_{j}$ in the prime variables (cf. (1.4)).

Remark 1.2. Whatever the ordered set $J$, for all $j \in J$ and for all $z, s, z^{\prime}$, $s^{\prime}, \sigma$ we have, modulo $d z^{\prime} \wedge d w^{\prime} \wedge d z \wedge d w$, the following identities:

$D a_{j}=\sum_{i=1}^{n} L_{i} a_{j} d \bar{z}_{i}+L_{i}^{\prime} a_{j} d \bar{z}_{i}^{\prime}$,

$D\left(D a_{J \backslash j}\right)=0$,

$D\left(i \sigma\left[s-s^{\prime}+i \varphi(z, s)-i \varphi\left(z^{\prime}, s^{\prime}\right)\right]\right)=0$.

We still need some more notation. In all that follows we assume $z, z^{\prime} \in$ $\Omega \odot \mathbf{C}^{n} ; s, s^{\prime} \in \mathscr{T} \odot \mathbf{R}^{d}$. The integrals over $\mathscr{O}:=\Omega \times \mathscr{T} \subset \mathscr{U}$ shall be 
calculated in the prime-variables $z^{\prime}, s^{\prime}$ and we make the following convention about the sign:

$$
\begin{aligned}
\int_{\mathscr{O}} f_{J}\left(z^{\prime}, s^{\prime}\right) d \bar{z}^{\prime} \wedge d z^{\prime} \wedge d w^{\prime} \wedge d \bar{z}_{J_{1}} \wedge d z_{J_{2}} \wedge d w_{J_{3}} \\
\stackrel{\text { def }}{=}(-1)^{(m+n) \cdot\left(\left|J_{1}\right|+\left|J_{2}\right|+\left|J_{3}\right|\right)} \\
\cdot\left(\int_{\mathscr{O}} f_{J}\left(z^{\prime}, s^{\prime}\right) d \bar{z}^{\prime} \wedge d z^{\prime} \wedge d w^{\prime}\right) d \bar{z}_{J_{1}} \wedge d z_{J_{2}} \wedge d w_{J_{3}} .
\end{aligned}
$$

In other words, we suppose the primed forms $d \bar{z}^{\prime} \wedge d z^{\prime} \wedge d w^{\prime}$ to be on the right side before doing the integration. While speaking of the sign: we suppose $\mathbf{C}^{n}$ is oriented in such a way that the volume form is $d V=d x_{1} \wedge d y_{1} \wedge \cdots \wedge d x_{n} \wedge d y_{n}=$ $(-1)^{\left(\begin{array}{l}n \\ 2\end{array}\right)}(2 i)^{-n} d \bar{z} \wedge d z$.

We shall write $\varepsilon_{(1, \ldots, n)}^{J J^{*}}, \varepsilon_{J}^{j J \backslash j}$, etc. $\in\{1,-1\}$ for the sign of the permutations $(1, \ldots, n) \mapsto J J^{*}, J \mapsto j J \backslash j$ etc. respectively. To shorten the formulas we use $\lambda:=\left(z-z^{\prime}\right), D \lambda:=D \lambda_{1} \wedge \cdots \wedge D \lambda_{n}, D \bar{\lambda}_{J}:=D \bar{\lambda}_{j_{1}} \wedge \cdots \wedge D \bar{\lambda}_{j_{v+1}}$, etc.

1.2. The formula. We shall need the Newtonian potential. We write

$$
\forall_{1 \leq j \leq n} \forall_{0 \leq \nu \leq n-1} \quad \mathscr{E}_{j}^{(\nu)}(\lambda):=(-1)^{\nu}(n-\nu-1) ! \frac{\bar{\lambda}_{j}}{|\lambda|^{2(n-\nu)}}
$$

and define a form $E_{a}=E_{a}\left(z, s, z^{\prime}, s^{\prime}, \sigma\right)$ in

$$
C^{\infty}\left(\mathscr{U} \times \mathscr{U} \backslash\left\{z=z^{\prime}\right\} \times \mathbf{R}_{d}, \Lambda^{2 n-1}\right) \cap L_{\mathrm{loc}}^{1}\left(\mathscr{U} \times \mathscr{U} \times \mathbf{R}_{d}, \Lambda^{2 n-1}\right)
$$

as follows:

$$
E_{a}:=\sum_{\nu=0}^{n-1} \sum_{|J|=\nu+1} \sum_{j \in J} \varepsilon_{J}^{j J \backslash j} \varepsilon_{(1, \ldots, n)}^{J J^{*}} \mathscr{E}_{j}^{(\nu)}(\lambda) D a_{J \backslash j} \wedge D \bar{\lambda}_{J^{*}} \wedge D \lambda .
$$

As before, we assume $0 \leq q \leq n$ and $\mathscr{O}=\Omega \times \mathscr{T} \odot \mathscr{U} \subset \mathbf{C}^{n} \times \mathbf{R}^{d}$.

Definition 1.3. Let us write $[\cdots]$ for $\left[s-s^{\prime}+i \varphi(z, s)-i \varphi\left(z^{\prime}, s^{\prime}\right)\right]$ in the following equations. For a current $f\left(z^{\prime}, s^{\prime}\right)=\sum_{|J|=q} f_{J}\left(z^{\prime}, s^{\prime}\right) d \bar{z}_{j}^{\prime} \wedge d z^{\prime} \wedge d w^{\prime} \in$ $\mathscr{D}^{\prime}\left(\Sigma, \Lambda^{m, q}\right)$ we set

$$
\begin{aligned}
& \mathscr{K}_{\mathscr{O}}^{a, \varphi} f(z, s, \sigma):=(2 i \pi)^{-n} \int_{\mathscr{O}} e^{i \sigma[\cdots]+a\left(z-z^{\prime}\right)} f\left(z^{\prime}, s^{\prime}\right) \wedge E_{a} \wedge d w, \\
& \mathscr{K}_{\partial \mathscr{O}}^{a, \varphi} f(z, s, \sigma):=(2 i \pi)^{-n} \int_{\partial \mathscr{O}} e^{i \sigma[\cdots]+a\left(z-z^{\prime}\right)} f\left(z^{\prime}, s^{\prime}\right) \wedge E_{a} \wedge d w, \\
& \mathscr{R}_{\mathscr{O}}^{a, \varphi} f(z, s, \sigma):=(2 i \pi)^{-n}(-1)^{d} \int_{\mathscr{O}} e^{i \sigma[\cdots]+a\left(z-z^{\prime}\right)} f\left(z^{\prime}, s^{\prime}\right) \wedge D a \wedge D \lambda \wedge d w, \\
& \mathscr{J}^{\varphi} f_{J}(z, s, \sigma):=(-1)^{\left(\begin{array}{c}
n+1 \\
2
\end{array}\right)} \int_{\mathscr{T}} e^{i \sigma\left[s-s^{\prime}+i \varphi(z, s)-i \varphi\left(z, s^{\prime}\right)\right]} f_{J}\left(z, s^{\prime}\right) \operatorname{det}\left(1+i \varphi_{s}\right) d s^{\prime} .
\end{aligned}
$$

We note that $\mathscr{K}_{\mathscr{O}} f \in \mathscr{D}^{\prime}\left(\Sigma, \Lambda^{m, q-1}\right)$, and in particular $\mathscr{K}_{\mathscr{O}} f=0$ in the case $q=0$. The currents $\mathscr{K}_{\partial \mathscr{O}} f, \mathscr{R}_{\mathscr{O}} f$ and $\mathscr{I}^{\varphi} f$ belong to $\mathscr{D}^{\prime}\left(\Sigma, \Lambda^{m, q}\right)$.

Theorem 1.4 (Bochner-Martinelli-Koppelman-Trèves). Let $0 \leq q \leq n$. For any differential form $f=\sum_{|J|=q} f_{J} d \bar{z}_{J}^{\prime} \wedge d z^{\prime} \wedge d w^{\prime} \in C^{1}\left(\Sigma, \Lambda^{m, q}\right)$, the following identity holds:

$$
\mathscr{I}^{\varphi} f=d\left((-1)^{q} \mathscr{K}_{\mathscr{O}}^{a, \varphi} f\right)+(-1)^{q+1} \mathscr{K}_{\mathscr{O}}^{a, \varphi} d f+\mathscr{R}_{\mathscr{O}}^{a, \varphi} f+(-1)^{q} \mathscr{K}_{\partial \mathscr{\theta}}^{a, \varphi} f .
$$

For a proof of this formula, see [T3] or [P]. 
1.3. The error term. Still following [T3], we now give a condition that guarantees the vanishing of the error term in our homotopy formula. Let us recall the notation (1.1) and put

$$
L_{j}^{\prime}:=\frac{\partial}{\partial \bar{z}_{j}^{\prime}}-i^{t} \varphi_{\bar{z}_{j}^{\prime}}{ }^{t}\left(1+i \varphi_{s^{\prime}}\right)^{-1}{ }^{t} \partial_{s^{\prime}}, \quad \text { for } 1 \leq j \leq n .
$$

Theorem 1.5. Let $0 \leq q \leq n$ and again $f=\sum_{|J|=q} f_{J} d \bar{z}_{J}^{\prime} \wedge d z^{\prime} \wedge d w^{\prime} \in$ $\mathscr{D}^{\prime}\left(\Sigma, \Lambda^{m, q}\right)$. If $\operatorname{rank}\left(L_{j} a_{i}\right)<q$ or $\operatorname{rank}\left(L_{j}^{\prime} a_{i}\right)<n-q$, then $\mathscr{R}_{\mathscr{\theta}}^{a, \varphi} f=0$.

Proof. $\mathscr{R}_{\mathscr{O}} f$ is of the form $\sum_{|J|=q} \int_{\mathscr{O}} e f_{J} d \bar{z}_{J}^{\prime} \wedge d z^{\prime} \wedge d w^{\prime} \wedge D a \wedge d z \wedge d w$ where the $e f_{J}$ are functions. By Remark 1.2 we can replace $D a=\bigwedge_{k=1}^{n} D a_{k}$ by $\bigwedge_{k=1}^{n} \sum_{j=1}^{n}\left(L_{j} a_{k} d \bar{z}_{j}+L_{j}^{\prime} a_{k} d \bar{z}_{j}^{\prime}\right)$. But in the integral over $\mathscr{O}$ we need $q$ terms in $d \bar{z}_{j}$ and $n-q$ terms in $d \bar{z}_{j}^{\prime}$. Up to signs, we therefore have

$$
\begin{aligned}
\mathscr{R}_{\mathscr{O}} f=\sum_{|J|=q} \sum_{|K|=q}\left(\int_{\mathscr{O}} e f_{J} \operatorname{det}\left(L_{j}^{\prime} a_{k}\right)_{j \in J^{*}, k \in K^{*}} d \bar{z}^{\prime} \wedge d z^{\prime} \wedge d w\right) \\
\cdot \operatorname{det}\left(L_{j} a_{k}\right)_{j \in J, k \in K} d \bar{z}_{K} \wedge d z \wedge d w .
\end{aligned}
$$

Our hypothesis implies that each term in this sum is zero.

\section{The CASE OF A REAL HYPERSURFACE $\Sigma$ IN $\mathbf{C}^{n+1}$}

2.1. The problem. We want to study the local hypoellipticity of the CRstructure on $\mathscr{M}$ in the case of codimension $d=1$. As before we use the local embedding $p$ and work with the real hypersurface $p(\mathscr{M})=\Sigma \subset \mathbf{C}^{n+1}$.

Let $q$ be another integer and, from now on, let $\mathscr{D}^{\prime}\left(\Sigma, \Lambda^{n+1, q}\right)$ denote the set of germs of currents of bidegree $(n+1, q)$ defined at the point $0 \in \Sigma$. The goal of this section is to find sufficient conditions for the structure to satisfy the following property.

Definition 2.1. $\bar{\partial}_{\Sigma}$ is called q-hypoelliptic if for all $v \in \mathscr{D}^{\prime}\left(\Sigma, \Lambda^{n+1, q}\right)$ the following holds: $\bar{\partial}_{\Sigma} v$ smooth $\Rightarrow \exists u \in \mathscr{D}^{\prime}\left(\Sigma, \Lambda^{n+1, q-1}\right)$ such that $v-\bar{\partial}_{\Sigma} u$ is smooth. (We put $\Lambda^{n+1, q}=0$ if $q<0$ or $q>n$.)

Remark. $\mathscr{D}^{\prime}$-local solvability in dimension $q$ implies $q$-hypoellipticity.

Example. Let $\Sigma=\{\operatorname{Im} w=0\}$ be a hyperplane and $f=\sum f_{J} d \bar{z}_{J} \wedge d z \wedge d w \in$ $\mathscr{D}^{\prime}\left(\Sigma, \Lambda^{n+1, q}\right)$. Then $\bar{\partial}_{\Sigma}$ is given by

$$
\bar{\partial}_{\Sigma} f=\sum_{j=1}^{n} \sum_{|J|=q} \frac{\partial f_{J}}{\partial \bar{z}_{j}} d \bar{z}_{j} \wedge d \bar{z}_{J} \wedge d z \wedge d w .
$$

Let $v=\left|z_{1}\right| d \bar{z}_{1} \wedge d z \wedge d w \in \mathscr{D}^{\prime}\left(\Sigma, \Lambda^{n+1,1}\right)$. Then we have $\bar{\partial}_{\Sigma} v$ smooth (zero!) although $v$ is not. But $v$ is exact, namely $v=\bar{\partial}_{\Sigma}\left(\frac{2}{3} \bar{z}_{1}\left|z_{1}\right| d z \wedge d w\right)$. In fact, for any $1 \leq q \leq n, \bar{\partial}_{\Sigma}$ is $q$-hypoelliptic since for any current $v=$ $f(z, s) d \bar{z}_{J} \wedge d z \wedge d w$, we can use the Cauchy formula to solve $\partial u_{0} / \partial \bar{z}_{j_{1}}=f$ and write, letting $I:=J \backslash j_{1}, u=u_{0} d \bar{z}_{I} \wedge d z \wedge d w$, such that

$$
\bar{\partial}_{\Sigma} u=v+\sum_{j \notin J} \frac{\partial u_{0}}{\partial \bar{z}_{j}} d \bar{z}_{j} \wedge d \bar{z}_{I} \wedge d z \wedge d w
$$


The $\partial u_{0} / \partial \bar{z}_{j}$ are smooth once $\bar{\partial}_{\Sigma} v$ is smooth.

On the other hand, $\bar{\partial}_{\Sigma}$ is not 0 -hypoelliptic, because if $v=|s| d z \wedge d w$ then we have $\bar{\partial}_{\Sigma} v=0$, while $v$ is not cohomologous to a smooth form.

2.2. Microlocalisation. The characteristic set $T^{0}$ of the CR-structure on $\mathscr{M}$ is the subset of $T^{*} \mathscr{M} \backslash 0$ where the symbol of the operator $\bar{\partial}_{\Sigma}$ vanishes. By (1.1)-(1.2) we have

$$
\begin{aligned}
T^{0} & =\text { Char } \bar{\partial}_{\Sigma} \\
& =\left\{(z, s, \zeta, \sigma) \mid(\zeta, \sigma) \neq(0,0), \forall_{j=1, \ldots, n} \text { symb }^{j}=0\right\},
\end{aligned}
$$

where

$$
\mathrm{symb}^{j}=\bar{\zeta}^{j}-i \varphi_{\bar{z}_{j}}(z, s) \frac{\sigma}{1+i \varphi_{s}(z, s)} .
$$

By Sato's theorem (cf. [T1, p. 70] or [H2, Theorem 8.3.1]), for all $q, \bar{\partial}_{\Sigma}$ is $q$-hypoelliptic outside of this set, i.e., for all germs of currents $v$ we have $\bar{\partial}_{\Sigma} v$ smooth $\Rightarrow \exists u$ such that $\operatorname{WF}\left(v-\bar{\partial}_{\Sigma} u\right) \subset T^{0}$. We want to find under which conditions $\bar{\partial}_{\Sigma}$ is $q$-hypoelliptic inside $T^{0}$.

We remark that $T^{0}$ is of rank $d=1$ and $\forall(z, s, \zeta, \sigma) \in T^{0}$ we have $\sigma \neq 0$.

Definition 2.2. Let us suppose an orientation for $T^{0}$ has been chosen such that we can write $T^{0}=:\{\sigma>0\} \sqcup\{\sigma<0\}$. We shall call the operator $\bar{\partial}_{\Sigma}$

- q-hypoelliptic ${ }^{+}$if for all $v \in \mathscr{D}^{\prime}\left(\Sigma, \Lambda^{n+1, q}\right)$ one has the following implication: $\bar{\partial}_{\Sigma} v$ smooth $\Rightarrow \exists u \in \mathscr{D}^{\prime}\left(\Sigma, \Lambda^{n+1, q-1}\right)$ such that $\operatorname{WF}\left(v-\bar{\partial}_{\Sigma} u\right)$ is contained in the negative part $\{\sigma<0\}$ of the characteristic set $T^{0}$.

- q-hypoelliptic ${ }^{-}$if each germ of current $v \in \mathscr{D}^{\prime}\left(\Sigma, \Lambda^{n+1, q}\right)$ with $\bar{\partial}_{\Sigma} v$ smooth is cohomologous to a smooth germ in the negative part $\{\sigma<0\}$ of the characteristic set.

Remark 2.3. No matter what orientation for $T^{0}$ has been chosen, $\bar{\partial}_{\Sigma}$ is $q$ hypoelliptic if and only if it is both $q$-hypoelliptic ${ }^{+}$and $q$-hypoelliptic ${ }^{-}$.

Proof. $\Rightarrow$ is trivial, $\Leftarrow$ follows from Sato's theorem.

2.3. The geometry of $\Sigma$. We shall always assume $0 \leq q \leq n$. (Otherwise $\bar{\partial}_{\Sigma}$ would be trivially $q$-hypoelliptic.) Let $/ \mathbf{\Lambda}: \mathbf{C}_{x}^{q} \times \Sigma \rightarrow \mathbf{C}^{n+1}$ be a smooth map, holomorphic in $x$, such that, for all $P \in \Sigma,(0, P) \stackrel{\mid \Lambda}{\mapsto} P$ and $\partial_{x}|/ \mathbf{\Lambda}|_{(0, P)}$ is of maximal rank. If furthermore the manifolds $/ \mathbf{\Lambda}_{P} \stackrel{\text { def }}{=}\left\{/ \mathbf{\Lambda}(x, P) \mid x \in \mathbf{C}^{q}\right\}$ are tangent to $\Sigma$ at $P$ for all points of $\Sigma$, then we shall call $\left\{/ \Lambda_{P}\right\}_{P \in \Sigma}$ a family of holomorphic supporting manifolds of dimension $q$.

We shall assume that the manifolds $\Lambda_{P}$ stay strictly on one side of the hypersurface $\Sigma$, touching it only at the point $P$. But as long as $q+q^{\prime} \leq n, \Sigma$ could allow holomorphic supporting manifolds of dimension $q$ on one side and of dimension $q^{\prime}$ on the other side simultaneously.

Example. $\Sigma=\left\{\left.(z, w) \in \mathbf{C}^{3}|\operatorname{Im} w=| z_{1}\right|^{2}-\left|z_{2}\right|^{2}\right\}$ admits one-dimensional holomorphic supporting manifolds on both sides. In fact, the map

$$
/ \mathbf{\Lambda}: \mathbf{C} \times \Sigma \rightarrow \mathbf{C}^{3} ; \quad(x, z, w) \mapsto\left(x+z_{1}, z_{2}, 2 i \bar{z}_{1} x+w\right)
$$

defines curves below $\Sigma$ :

$$
\forall P=(z, w) \in \Sigma \forall_{x \in \mathbf{C}} \operatorname{Im}\left\{2 i \bar{z}_{1} x+w\right\} \leq\left|x+z_{1}\right|^{2}-\left|z_{2}\right|^{2} .
$$




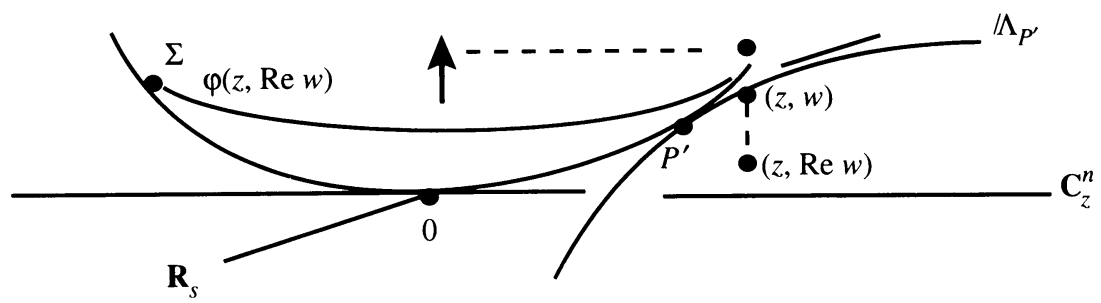

FIGURE 1

Similarly, the holomorphic curves in $x$ defined by

$$
/ \mathbf{\Lambda}: \mathbf{C} \times \Sigma \rightarrow \mathbf{C}^{3} ; \quad(x, z, w) \mapsto\left(z_{1}, x+z_{2},-2 i \bar{z}_{2} x+w\right)
$$

remain above $\Sigma$.

At the end of the present section, hopefully the reader will be convinced of the following result:

Theorem 2.4. If $\Sigma$ admits holomorphic supporting manifolds of dimension $q+1$ on both sides, then the operator $\bar{\partial}_{\Sigma}$ is q-hypoelliptic and $(n-q)$-hypoelliptic.

In the case of the preceding example, this theorem says that every distribution $u(z, s)$ solving the system

$$
\left.\begin{array}{l}
\partial_{\bar{z}_{1}} u-i z_{1} \partial_{s} u=\text { smooth } \\
\partial_{\bar{z}_{2}} u+i z_{2} \partial_{s} u=\text { smooth }
\end{array}\right\}
$$

is smooth. This result is well known (first proven in [H1, 2], also see [FS, He]), and we shall come back to it. Theorem 2.4 is a special case of the following one, which will distinguish between the two sides of $\Sigma$ and give a microlocal conclusion. We want to use the words "above" and "below" exactly as we did in the example, i.e., for any hypersurface given in coordinates such that $\Sigma=$ $\{\operatorname{Im} w=\varphi(z, \operatorname{Re} w)\}$ with $\varphi(0)=0, d \varphi(0)=0$, let us agree to measure the height by the coordinate $\operatorname{Im} w$.

Definition 2.5. The hypersurface $\Sigma=\left\{(z, w) \in \mathbf{C}^{n+1} \mid \operatorname{Im} w=\varphi(z, \operatorname{Re} w)\right\}$ is called $q$-holoconcave $u p$ if there exists a family $\left\{/ \mathbf{\Lambda}_{P}\right\}_{P \in \Sigma}$ of $q$-dimensional holomorphic supporting manifolds underneath it, i.e., such that for all $P^{\prime} \in$ $\boldsymbol{\Sigma}$ and for all $(z, w) \in / \Lambda_{P^{\prime}} \operatorname{Im} w<\varphi(z, \operatorname{Re} w)$. Similarly, $\Sigma$ is called $q$ holoconcave down if $\forall_{P^{\prime} \in \Sigma} \forall_{(z, w) \in / \Lambda_{P^{\prime}}} \operatorname{Im} w ? \varphi(z, \operatorname{Re} w)$. See Figure 1 .

In place of an example, let us assume $\Sigma$ to be rigid and put $q=1$. We then get the following result.

Proposition 2.6. A rigid hypersurface $\left.\Sigma=\{z, w) \in \mathbf{C}^{n+1} \mid \operatorname{Im} w=\varphi(z)\right\}$ is 1holoconcave up (down) if for all $P=(z, w) \in \Sigma$ the function $\mathbf{C} \rightarrow \mathbf{R} ; x \mapsto$ $\varphi\left(z_{1}+x, z_{2}, \ldots, z_{n}\right)$ is convex (concave).

Proof. Since $\left.\partial r\right|_{P}=d w / 2 i-\left.\sum_{j=1}^{n} \partial_{z_{j}} \varphi\right|_{z} d z^{j}$, the holomorphic curve $/ \Lambda_{P}(x):=$ $\left(z_{1}+x, z_{2}, \ldots, z_{n}, w+\left.2 i x \partial_{z_{1}} \varphi\right|_{z}\right)$ is contained in the complex tangent space $T_{P}^{\mathrm{C}} \Sigma$. By hypothesis, we have $\forall_{x \in \mathrm{C}} \varphi(z)+2 \operatorname{Re}\left\{\left.x \partial_{z_{1}} \varphi\right|_{z}\right\} \leqslant \varphi\left(z_{1}+x, z_{2}, \ldots, z_{n}\right)$. $\Lambda_{P}$ therefore remains below $\Sigma$.

Examples. 1. For any $\nu \in \mathbf{N}, \Sigma=\left\{\left.(z, w) \in \mathbf{C}^{3}|\operatorname{Im} w=| z_{1}\right|^{2 \nu}-\left|z_{2}\right|^{2 \nu}\right\}$ is 1-holoconcave up and 1-holoconcave down. 
2. $\Sigma=\left\{(z, w) \in \mathbf{C}^{3}|\operatorname{Im} w=| z_{1}-\left.z_{2}{ }^{3}\right|^{2}\right\}$ is 1-holoconcave up. In fact, by letting $z(u):=\left(u_{1}+u_{2}^{3}, u_{2}\right)$ we can define the holomorphic supporting manifolds from below as

$$
/ \mathbf{\Lambda}: \mathbf{C} \times \Sigma \rightarrow \mathbf{C}^{3} ; \quad(x, z(u), w) \mapsto\left(z\left(u_{1}+x, u_{2}\right), w+\left.2 i x \partial_{u_{1}}(\varphi \circ z)\right|_{u}\right) .
$$

We remark that in these examples the Levi form of the structure is degenerate at the origin.

\subsection{The theorem.}

Theorem 2.7. Let $0 \leq q \leq n$ and let $\Sigma$ be a real hypersurface of $\mathbf{C}^{n+1}$. Then the characteristic set $T^{0}$ can be oriented in such a way that the following implications hold: If $\Sigma$ is $q$-holoconcave down, then $\bar{\partial}_{\Sigma}$ is $q^{\prime}$-hypoelliptic ${ }^{+}$and $\left(n-q^{\prime}\right)$-hypoelliptic ${ }^{-}$, for all $q^{\prime}<q$. If $\Sigma$ is q-holoconcave up, then $\bar{\partial}_{\Sigma}$ is $q^{\prime}$-hypoelliptic ${ }^{-}$and $\left(n-q^{\prime}\right)$-hypoelliptic ${ }^{+}$as long as $q^{\prime}<q$.

Example $(n=2) . \Sigma=\left\{\left.(z, w) \in \mathbf{C}^{3}|\operatorname{Im} w=| z_{1}\right|^{2}+\left|z_{2}\right|^{2}\right\}$ is 2-holoconcave up. Consider once more the Lewy vector fields

$$
L_{j}:=\partial_{\bar{z}_{j}}-i z_{j} \partial_{s}, \quad \text { for } j=1,2 \text {. }
$$

Assume $v_{1}, v_{2}$ are distributions defined near $P \in \mathbf{C}^{2} \times \mathbf{R}$. If $L_{1} v_{2}-L_{2} v_{1}$ is smooth, then the theorem yields a distribution $u$ such that $v_{j}-L_{j} u$ is smooth in some neighbourhood of $P$, for $j=1,2$. Moreover, if both $L_{1} v$ and $L_{2} v$ are smooth for a distribution $v$, then the theorem says that WF $v \subset\{\sigma>0\}$, and for an arbitrary distribution $v$ and any point $P$ there always exist distributions $u_{1}, u_{2}$, so that near $P$ the wave front of $v-L_{1} u_{2}+L_{2} u_{1}$ is contained in the negative part of the characteristic set.

Corollary 2.8. Let $\Sigma$ be a 1-holoconcave up (down) real hypersurface in $\mathbf{C}^{n+1}$. Provided the characteristic set has the right orientation, the following holds true: If $u \in \mathscr{D}^{\prime}(\Sigma)$ is a distribution such that $\bar{\partial}_{\Sigma} u$ is smooth, then WF $u$ is contained in the positive (negative) part of the characteristic set.

Examples $(n=2)$. 1. $\Sigma=\left\{\left.(z, w) \in \mathbf{C}^{3}|\operatorname{Im} w=| z_{1}\right|^{2 \nu}-\left|z_{2}\right|^{2 \nu}\right\}$. Let $f=$ $u(z, s) d z \wedge d w$. Then

$$
\begin{aligned}
\bar{\partial}_{\Sigma} f=\left(\left(\partial_{\bar{z}_{1}} u-i \nu z_{1}\left|z_{1}\right|^{2 \nu-2} \partial_{s} u\right) d \bar{z}_{1}\right. \\
\left.+\left(\partial_{\bar{z}_{2}} u+i \nu z_{2}\left|z_{2}\right|^{2 \nu-2} \partial_{s} u\right) d \bar{z}_{2}\right) \wedge d z \wedge d w .
\end{aligned}
$$

The corollary says that each solution of the system (2.2) is smooth. One of these equations by itself allows nonsmooth solutions, however. For

$$
u(z, s):=\lim _{\varepsilon \downarrow 0} \frac{1}{2 \pi\left(i s-\left|z_{1}\right|^{2}-\varepsilon\right)}
$$

we have $\partial_{\bar{z}_{1}} u-i z_{1} \partial_{s} u=0$ and $u$ is not smooth in the plane $\left\{z_{1}=s=0\right\}$. But since $u=\mathscr{F}_{\sigma}^{-1}\left(e^{-\left|z_{1}\right|^{2} \sigma} H(\sigma)\right)$ we see that WF $u \subset\{\sigma>0\}$.

2. $\Sigma=\left\{(z, w) \in \mathbf{C}^{3}|\operatorname{Im} w=| z_{1}-\left.z_{2}{ }^{3}\right|^{2}\right\}$. Here $\varphi_{\bar{z}_{1}}=\left(z_{1}-z_{2}{ }^{3}\right), \varphi_{\bar{z}_{2}}=$ $-3 \bar{z}_{2}{ }^{2}\left(z_{1}-z_{2}{ }^{3}\right)$. The theorem says that each distribution $u(z, s)$ solving the system

$$
\left.\begin{array}{rl}
\partial_{\bar{z}_{1}} u-i\left(z_{1}-z_{2}{ }^{3}\right) \partial_{s} u & =\text { smooth } \\
\partial_{\bar{z}_{2}} u+3 i \bar{z}_{2}^{2}\left(z_{1}-z_{2}{ }^{3}\right) \partial_{s} u= & \text { smooth }
\end{array}\right\}
$$


is smooth in the negative part of the characteristic set. Similarly, each current $u \in \mathscr{D}^{\prime}\left(\Sigma, \Lambda^{2,2}\right)$ solving $(2.3)$ is cohomologous to a smooth form in $\{\sigma>0\}$.

3. Let us also look at a nonrigid example. Assume $\psi(s)$ is a differentiable real-valued function which takes the value 1 at the origin. Let $\mu, \nu$ be positive integers and $a, b$ be real numbers with $a b>0$. Suppose $u(z, s)$ is a distribution in $\mathbf{C}^{2} \times \mathbf{R}$ such that

$$
\begin{aligned}
& \partial_{\bar{z}_{1}} u-i a z_{1}\left|z_{1}\right|^{\mu-1} \psi(s) \partial_{s} u=\text { smooth }, \\
& \partial_{\bar{z}_{2}} u+i b z_{2}\left|z_{2}\right|^{\nu-1} \psi(s) \partial_{s} u=\text { smooth } .
\end{aligned}
$$

Then the corollary asserts that $u$ is smooth.

In fact, the two vector fields define the tangential Cauchy-Riemann operator on the manifold

$$
\Sigma=\left\{\left.(z, w) \in \mathbf{C}^{3}|\operatorname{Im} w=a| z_{1}\right|^{2 \mu}-b\left|z_{2}\right|^{2 \nu}+\tilde{\psi}(s)\right\}
$$

where the function $\tilde{\psi}$ is given by the equations $\tilde{\psi}^{\prime}(s)=1 / \psi(s)-1, \tilde{\psi}(0)=0$ in a neighbourhood of the origin. $\Sigma$ is 1-holoconcave up and 1-holoconcave down.

2.5. Proof. Let us assume $\Sigma=\left\{(z, w) \in \mathbf{C}^{n+1} \mid \operatorname{Im} w=\varphi(z, \operatorname{Re} w)\right\}$ is $q$ holoconcave up. In other words, we suppose the existence of a smooth map $\boldsymbol{\Lambda}: \mathbf{C}_{x}^{q} \times \Sigma \rightarrow \mathbf{C}^{n+1} ;\left(0, P^{\prime}\right) \mapsto P^{\prime}$, holomorphic in $x$, such that $\partial_{x} /\left.\mathbf{\Lambda}\right|_{0}$ is of maximal rank $q$ and $\forall_{P^{\prime} \in \Sigma}$ the $q$-manifold $/ \boldsymbol{\Lambda}_{P^{\prime}}=\left\{/ \boldsymbol{\Lambda}\left(x, P^{\prime}\right)\right\}$ remains below $\Sigma$ :

$$
\forall P^{\prime} \in \Sigma \forall_{x \in \mathbf{C}^{q}} \quad \operatorname{Im} / \mathbf{\Lambda}^{n+1}\left(x, P^{\prime}\right) \leq \varphi\left(\Lambda\left(x, P^{\prime}\right), \operatorname{Re} / \mathbf{\Lambda}^{n+1}\left(x, P^{\prime}\right)\right),
$$

where $\Lambda$ denotes the first $n$ components of $/ \Lambda$.

We need to show that under the hypotheses $\bar{\partial}_{\Sigma}$ is $\left(n-q^{\prime}\right)$-hypoelliptic ${ }^{+}$, for all $q^{\prime}<q$, but it suffices to do this for $q^{\prime}=q-1$ because our hypothesis gets weaker as $q$ decreases. Now let $f=\sum_{|J|=n-q+1} f_{J}(z, s) d \bar{z}_{J} \wedge d z \wedge d w \in$ $\mathscr{D}^{\prime}\left(\Sigma, \Lambda^{n+1, n-q+1}\right)$ be a current of bidegree $(n+1, n-q+1)$ such that $\bar{\partial}_{\Sigma} f$ is smooth. We claim that $f$ is cohomologous to a smooth form in the positive part $\{\sigma>0\}$ of the characteristic set.

Before we begin the proof, let us recall that always $z, z^{\prime} \in \Omega, s, s^{\prime} \in \mathscr{T}$, and $\mathscr{O}=\Omega \times \mathscr{T} \subset \subset \mathscr{U} \subset \mathbf{R}^{2 n+1}$.

These neighbourhoods of the origin will be shrunk as necessary during the proof. We shall continue to write, abusing notation, $\Sigma$ instead of $p(\mathscr{U})$. We shall always write $P=(z, w)=(z, s+i \varphi(z, s))$ and $P^{\prime}=\left(z^{\prime}, w^{\prime}\right)=\left(z^{\prime}, s^{\prime}+\right.$ $\left.i \varphi\left(z^{\prime}, s^{\prime}\right)\right) \in \Sigma$.

First step: Reduction to a $C^{1}$-form. Let

$$
L_{0}:=\frac{\partial_{s}}{1+i \varphi_{s}}
$$

and recall the definition of $L_{1}, \ldots, L_{n}$. The operator $\Delta_{L}=L_{0}^{2}+\cdots+L_{n}^{2}$ commutes with all $L_{j}$ 's and is elliptic (as long as we are close to zero). By distribution theory, we can write $f_{J} \equiv \Delta_{L}^{K} \dot{f}_{J}\left(\bmod C^{\infty}\right)$ with $\dot{f}_{J} \in C^{1}(\Sigma)$, provided the number $K$ is big enough. Since we assume that, for all $I$, $\sum_{\{j\} \cup J=I} L_{j} f_{J} \equiv \sum \Delta_{L}^{K} L_{j} \dot{f}_{J}$ is smooth, we must have $\sum L_{j} \dot{f}_{J}$ smooth. It is therefore sufficient to prove our theorem for the $C^{1}$-form $\dot{f}$.

Henceforth we shall assume that $f \in C^{1}\left(\Sigma, \Lambda^{n+1, n-q+1}\right)$. 
Second step: Construction of $\alpha$ and $\mu$. Let $P^{\prime}$ be an arbitrary point on the real hypersurface $\Sigma$. Our holoconvexity hypothesis will give us a full complex supporting manifold for $\Sigma$, i.e., a complex hypersurface in $\mathbf{C}^{n+1}, \mathbf{L}_{P^{\prime}}:=\{(z, w) \in$ $\left.\mathbf{C}^{n+1} \mid w=w^{\prime}+H\left(z, P^{\prime}\right)\right\}$ below $\Sigma$, which does not touch $\Sigma$ anywhere except in $P^{\prime}$. We shall have enough information about the function $H$ to deduce the following

Lemma 2.9. There exist maps $\alpha: \mathscr{U} \times \mathscr{U} \rightarrow \mathbf{C}_{n}$ and $\mu: \mathscr{U} \times \mathscr{U} \rightarrow \mathbf{C}$ such that $\operatorname{Re} \mu \equiv 1$, for all points $P, P^{\prime} \in \Sigma \operatorname{Im}\left\{\mu\left[w-w^{\prime}-i \alpha\left(z-z^{\prime}\right)\right]\right\} ? 0$ and $\operatorname{rank}\left(L_{j} \alpha_{i}\right)_{i \leq i, j \leq n} \leq n-q$.

Example $(n=2, q=1)$. For $\varphi=\left|z_{1}\right|^{2}-\left|z_{2}\right|^{2}$ it is possible to choose $\alpha=$ $\left(2 \bar{z}_{1}^{\prime},-2 \bar{z}_{2}\right)$ and $\mu=1+i\left(s-s^{\prime}+2 \operatorname{Im}\left\{\bar{z}_{1}^{\prime} z_{1}+\bar{z}_{2} z_{2}^{\prime}\right\}\right)$.

Let $P^{\prime}=\left(z^{\prime}, w^{\prime}\right) \in \Sigma$ be an arbitrary point. We first manufacture a complex hypersurface $L_{P^{\prime}} \subset \mathbf{C}^{n+1}$ below $\Sigma$ using the $q$-manifolds $/ \Lambda_{P}$ for $P$ moving along an $(n-q)$-manifold $/ \Lambda_{P^{\prime}}^{*} \subset \Sigma$ transverse to $/ \Lambda_{P^{\prime}}$.

After renumbering the $z$-coordinates, we can assume the matrix $\partial_{x} /\left.\mathbf{\Lambda}^{1, \ldots, q}\right|_{0}$ is invertible. We recall that $w^{\prime}=s^{\prime}+i \varphi\left(z^{\prime}, s^{\prime}\right)$ and write $z=\left(z_{(1)}, z_{(2)}\right) \in$ $\mathbf{C}^{q} \times \mathbf{C}^{n-q}$.

We define for $y=\left(y_{q+1}, \ldots, y_{n}\right) \in \mathbf{C}^{n-q}$

$$
\begin{aligned}
s^{*}(y):= & s^{\prime}-2 \operatorname{Re}\left\{\frac{i \varphi_{\bar{z}_{(2)}}\left(z^{\prime}, s^{\prime}\right)}{1+i \varphi_{s}\left(z^{\prime}, s^{\prime}\right)}\left(\bar{y}-\bar{z}_{(2)}^{\prime}\right)\right\} \\
& +2 \operatorname{Re}\left\{\sum_{q+1 \leq j, k \leq n} c_{j k}\left(z^{\prime}, s^{\prime}\right)\left(\bar{y}_{j}-\bar{z}_{j}^{\prime}\right)\left(\bar{y}_{k}-\bar{z}_{k}^{\prime}\right)\right\}
\end{aligned}
$$

with

$$
c_{j k}\left(z^{\prime}, s^{\prime}\right)=\left.\frac{i \varphi_{s s} \varphi_{\bar{z}_{j}} \varphi_{\bar{z}_{k}}-\left(1+i \varphi_{s}\right)\left(\varphi_{s \bar{z}_{j}} \varphi_{\bar{z}_{k}}+\varphi_{s \bar{z}_{k}} \varphi_{\bar{z}_{j}}\right)-\left(1+i \varphi_{s}\right)^{2} \varphi_{\bar{z}_{j} \bar{z}_{k}}}{\left(1+i \varphi_{s}\right)^{3}}\right|_{z^{\prime}, s^{\prime}}
$$

and

$$
/ \mathbf{\Lambda}_{P^{\prime}}^{*}: \mathbf{C}^{n-q} \rightarrow \Sigma ; \quad y \mapsto\left(z_{(1)}^{\prime}, y, s^{*}+i \varphi\left(z_{(1)}^{\prime}, y, s^{*}\right)\right) .
$$

Note that $/ \Lambda_{P^{\prime}}^{*}\left(z_{(2)}^{\prime}\right)=P^{\prime}$. We define now the complex hypersurface $L_{P^{\prime}}:=$ $\bigcup_{P \in / \Lambda_{P^{\prime}}} / \Lambda_{P}$. From the construction, we see that $L_{P^{\prime}}$ lies underneath $\Sigma$ and touches it at precisely the points $P \in / \Lambda_{P^{\prime}}^{*}$.

Example. $\varphi=\left|z_{1}\right|^{2}-\left|z_{2}\right|^{2}, s^{*}(y)=s^{\prime}-2 \operatorname{Im}\left\{z_{2}^{\prime} \bar{y}\right\}$. (Here the second derivatives are zero.)

The idea is to bend $L_{P^{\prime}}$ away from $\Sigma$ along $/ \Lambda_{P^{\prime}}^{*}$. In order to do this, we seek a parametrization for $L_{P^{\prime}}$.

Let $z: \mathbf{C}^{q} \times \mathbf{C}^{n-q} \times \Sigma ;\left(u, P^{\prime}\right) \mapsto \Lambda\left(u_{(1)}-z_{(1)}^{\prime}, / \mathbf{\Lambda}_{P^{\prime}}^{*}\left(u_{(2)}\right)\right)$. We remark that

$$
\forall_{u_{(2)} \in \mathbf{C}^{n-q}, \forall_{P^{\prime} \in \Sigma}} \quad z\left(z_{(1)}^{\prime}, u_{(2)}, P^{\prime}\right)=\left(z_{(1)}^{\prime}, u_{(2)}\right) .
$$

This implies that the matrix

$$
\left.\partial_{u} z\right|_{z^{\prime}, P^{\prime}}=\left(\begin{array}{cc}
\partial_{x} /\left.\mathbf{\Lambda}^{1, \ldots, q}\right|_{0, P^{\prime}} & 0 \\
* & 1
\end{array}\right)
$$


is invertible as long as $P^{\prime}$ stays close to 0 . When we take into account the fact that the map $z$ is holomorphic in $u_{(1)}$ we see from this that also the real Jacobian is invertible at the origin, that is, $\left.\partial_{u}^{\mathbf{R}} z\right|_{0} \in \mathrm{Gl}_{2 n}(\mathbf{R})$. Therefore, after shrinking $\Omega$ and $\mathscr{T}$ as much as necessary, we can find an open neighbourhood of zero $\widehat{\Omega} \subset \mathbf{C}^{n} \times \Sigma$ such that the following holds true.

Remark 2.10. The map $\mathscr{Z}: \widehat{\Omega} \rightarrow \Omega \times \Sigma ;\left(u, P^{\prime}\right) \mapsto\left(z\left(u, P^{\prime}\right), P^{\prime}\right)$ is a diffeomorphism. Its first component $z$ is holomorphic in $u_{(1)}$ and satisfies the relations $\left.\partial_{u}^{\mathbf{R}} z\right|_{z^{\prime}, P^{\prime}} \in \mathrm{Gl}_{2 n}(\mathbf{R}), z\left(z^{\prime}, P^{\prime}\right)=z^{\prime},\left.\partial_{\bar{u}_{(2)}} z\right|_{z^{\prime}, P^{\prime}}=0$ and $\left.\partial_{\bar{u}_{(2)} \bar{u}_{(2)}}^{2} z\right|_{z^{\prime}, P^{\prime}}=$ 0 .

Our first goal is achieved: we can write $L_{P^{\prime}}$ in parameters as follows.

$$
L_{P^{\prime}}=\left\{\left(z\left(u, P^{\prime}\right), / \mathbf{\Lambda}^{n+1}\left(u_{(1)}-z_{(1)}^{\prime}, / \mathbf{\Lambda}_{P^{\prime}}^{*}\left(u_{(2)}\right)\right)\right) \in \mathbf{C}^{n+1} \mid\left(u, P^{\prime}\right) \in \widehat{\Omega}\right\} .
$$

It is not difficult to bend this surface away from $\Sigma$. In fact, we introduce the smooth maps

$$
\widehat{H}: \widehat{\Omega} \rightarrow \mathbf{C} ; \quad\left(u, P^{\prime}\right) \mapsto\left|\mathbf{\Lambda}^{n+1}\left(u_{(1)}-z_{(1)}^{\prime}, / \mathbf{\Lambda}_{P^{\prime}}^{*}\left(u_{(2)}\right)\right)-w^{\prime}-i\right| u_{(2)}-\left.z_{(2)}^{\prime}\right|^{2}
$$

and

$$
H: \Omega \times \Sigma \rightarrow \mathbf{C} ; \quad\left(z, P^{\prime}\right) \mapsto \widehat{H} \circ \mathscr{Z}^{-1}\left(z, P^{\prime}\right)
$$

and make the following

Definition 2.11. $\mathbf{L}_{P^{\prime}}:=\left\{(z, w) \in \mathbf{C}^{n+1} \mid w=w^{\prime}+H\left(z, P^{\prime}\right)\right\}$.

From the construction, it is clear that $\mathbf{L}_{P^{\prime}}$ lies strictly below $\Sigma$, touching it at the point $P^{\prime}$ only. In other words, (2.4) yields the following inequality:

$$
\begin{gathered}
\forall_{P^{\prime}=\left(z^{\prime}, w^{\prime}\right) \in \Sigma}, \quad \forall_{z=z\left(u, P^{\prime}\right) \in \Omega}, \\
\operatorname{Im}\left\{w^{\prime}+H\left(z, P^{\prime}\right)\right\} \leqslant \varphi\left(z, s^{\prime}+\operatorname{Re} H\left(z, P^{\prime}\right)\right)-\frac{1}{2}\left|u_{(2)}-z_{(2)}^{\prime}\right|^{2} .
\end{gathered}
$$

The inequality is strict except when $z=z^{\prime}$.

Remark 2.12. The function $\widehat{H}\left(u, P^{\prime}\right)=H\left(z\left(u, P^{\prime}\right), P^{\prime}\right)$ is holomorphic in $u_{(1)}$ and satisfies the identities $\widehat{H}\left(z^{\prime}, P^{\prime}\right)=H\left(z^{\prime}, P^{\prime}\right)=0,\left.\partial_{\bar{u}_{(2)}} \widehat{H}\right|_{z^{\prime}, P^{\prime}}=$ $\left.\partial_{\bar{z}_{(2)}} H\right|_{z^{\prime}, P^{\prime}}=0$ and, for all $q+1 \leq j, k \leq n,\left.\partial_{\bar{u}_{j} \bar{u}_{k}}^{2} \widehat{H}\right|_{z^{\prime}, P^{\prime}}=0$, whatever the point $P^{\prime}=\left(z^{\prime}, w^{\prime}\right) \in \Sigma$.

Proof. $H$ inherits the holomorphy in $u_{(1)}$ from $\Lambda$. The first identity follows directly from the definitions. The second one follows from (2.7) because

$$
\begin{aligned}
& \left.\partial_{\bar{u}_{(2)}} \widehat{H}\right|_{z^{\prime}, P^{\prime}}=\partial_{z} H \underbrace{\partial_{\left.\bar{u}_{(2)} z\right|_{z^{\prime}, P^{\prime}}}}_{\stackrel{(2.7)}{=} 0}+\partial_{\bar{z}_{(1)}} H \underbrace{\partial_{\bar{u}_{(2)}} \bar{z}_{(1)} \mid z_{z^{\prime}, P^{\prime}}}_{\stackrel{(2.7)}{=} 0} \\
& +\partial_{\bar{z}_{(2)}} H \underbrace{\partial_{\bar{u}_{(2)}} \bar{z}_{(2)}||_{z^{\prime}, P^{\prime}}}_{(2,7)}=\left.\partial_{\bar{z}_{(2)}} H\right|_{z^{\prime}, P^{\prime}} .
\end{aligned}
$$

The fact that these gradients as well as the second derivatives in $\bar{u}_{(2)}$ vanish at the points $\left(z^{\prime}, P^{\prime}\right) \in \widehat{\Omega}$ follows from our choice of the function $s^{*}\left(u_{(2)}\right)$, 
because thanks to $(2.6)$ we have

$$
\begin{aligned}
\left.\partial_{\bar{u}_{(2)}} \widehat{H}\right|_{z^{\prime}, P^{\prime}}= & \left.\partial_{\bar{u}_{(2)}}\left(s^{*}+i \varphi\left(z_{(1)}^{\prime}, u_{(2)}, s^{*}\right)\right)\right|_{u_{(2)}=z_{(2)}^{\prime}} \\
= & \left(1+i \varphi_{s}\right) \partial_{\bar{u}_{(2)}} s^{*}+\left.i \varphi_{\bar{z}_{(2)}}\right|_{z^{\prime}, s^{\prime}} \stackrel{(2.6)}{=} 0 \\
\left.\partial_{\bar{u}_{j} \bar{u}_{k}}^{2} \widehat{H}\right|_{z^{\prime}, P^{\prime}}= & \left.\partial_{\bar{u}_{j} \bar{u}_{k}}^{2}\left(s^{*}+i \varphi\left(z_{(1)}^{\prime}, u_{(2)}, s^{*}\right)\right)\right|_{z_{(2)}^{\prime}} \\
= & \left.\partial_{\bar{u}_{j}}\left(\left(1+i \varphi_{s}\right) \partial_{\bar{u}_{k}} s^{*}+i \varphi_{\bar{z}_{k}}\right)\right|_{z^{\prime}, s^{\prime}} \\
= & \left(i \varphi_{s s} \partial_{\bar{u}_{j}} s^{*}+i \varphi_{s \bar{z}_{j}}\right) \partial_{\bar{u}_{k}} s^{*}+\left(1+i \varphi_{s}\right) \partial_{\bar{u}_{j} \bar{u}_{k}}^{2} s^{*} \\
& +i \varphi_{\bar{z}_{j} \bar{z}_{k}}+\left.i \varphi_{s \bar{z}_{k}} \partial_{\bar{u}_{j}} s^{*}\right|_{z^{\prime}, s^{\prime}} \stackrel{(2.6)}{=} 0 .
\end{aligned}
$$

Remarks 2.10 and 2.12 imply the existence of a map $\widehat{\alpha}: \widehat{\Omega} \rightarrow \mathbf{C}_{n}$ such that

$$
\forall_{\left(u, P^{\prime}\right) \in \widehat{\Omega}} \widehat{H}\left(u, P^{\prime}\right)=i \widehat{\alpha}\left(u, P^{\prime}\right)\left(z\left(u, P^{\prime}\right)-z^{\prime}\right)+O\left(\left|u_{(2)}-z_{(2)}^{\prime}\right|^{3}\right) .
$$

From here it is easy to prove our Lemma 2.9, as we shall now see.

Definition 2.13. Let $M>0$ be greater than all second derivatives of $\varphi$ in $\mathscr{U}$. We then put $\alpha\left(z, P^{\prime}\right):=\widehat{\alpha} \circ \mathscr{Z}^{-1}\left(z, P^{\prime}\right)$ and $\mu\left(P, P^{\prime}\right):=1-i \varphi_{s}(z, s)+$ $i M\left(s-s^{\prime}-\operatorname{Re}\left\{i \alpha\left(z, P^{\prime}\right)\left(z-z^{\prime}\right)\right\}\right)$, for any $P=(z, s), P^{\prime}=\left(z^{\prime}, s^{\prime}\right) \in \Sigma$.

We note that (2.9) implies

$$
\forall P^{\prime} \in \Sigma \forall_{z=z\left(u, P^{\prime}\right) \in \Omega} \quad H\left(z, P^{\prime}\right)=i \alpha\left(z-z^{\prime}\right)+O\left(\left|u_{(2)}-z_{(2)}^{\prime}\right|^{3}\right)
$$

which will yield the first inequality in Lemma 2.9 .

In fact, let us write $\alpha:=\alpha\left(z, P^{\prime}\right)$ and $\mu:=\mu\left(z, s, z^{\prime}, s^{\prime}\right)$ for brevity, and put $\tilde{s}:=s^{\prime}+\operatorname{Re}\left\{i \alpha\left(z-z^{\prime}\right)\right\}$. Then we have

$$
\begin{aligned}
& \operatorname{Im}\left\{\mu\left[w-w^{\prime}-i \alpha\left(z-z^{\prime}\right)\right]\right\}=\operatorname{Im} \mu(s-\tilde{s})+\varphi(z, s)-\varphi(z, \tilde{s}) \\
& \quad+\varphi(z, \tilde{s})-\varphi\left(z, s^{\prime}+\operatorname{Re} H\left(z, P^{\prime}\right)\right) \\
& \quad+\varphi\left(z, s^{\prime}+\operatorname{Re} H\left(z, P^{\prime}\right)\right)-\operatorname{Im}\left\{w^{\prime}+H\left(z, P^{\prime}\right)\right\} \\
& \quad+\operatorname{Im} H\left(z, P^{\prime}\right)-\operatorname{Im}\left\{i \alpha\left(z-z^{\prime}\right)\right\} .
\end{aligned}
$$

We must show that this is strictly positive, except when $P=P^{\prime}$. The term (1) is $>0$ by Taylor's theorem, and vanishes iff $s=\tilde{s}$. The term (3) is $>\frac{1}{2}\left|u_{(2)}-z_{(2)}^{\prime}\right|^{2}$ by holoconvexity (2.8), and vanishes iff $z=z^{\prime}$. Now since $\tilde{s}-\left(s^{\prime}+\operatorname{Re} H\left(z, P^{\prime}\right)\right)=O\left(\left|u_{(2)}-z_{(2)}^{\prime}\right|^{3}\right)$ by $(2.10)$ and $\left.d \varphi\right|_{0}=0$ we see that the absolute value of (2) is strictly smaller than $\frac{1}{4}\left|u_{(2)}-z_{(2)}^{\prime}\right|^{2}$, unless it is zero. This same is true for the term (4), always provided we stay close to the origin. Thus we see that (2) $+(3)+(4)>0$, and zero iff $z=z^{\prime}$. The sum of all four terms is positive and vanishes only when $s=\tilde{s}$ and $z=z^{\prime}$, that is, precisely when $P=P^{\prime}$.

Finally, we use the fact that $\widehat{\alpha}$ is holomorphic in $u_{(1)}$ to show the second inequality in Lemma 2.9. Since $\alpha$ does not depend on $s$, we have for all $1 \leq j$, $k \leq n \quad L_{j} \alpha_{k}\left(P, P^{\prime}\right)=\left.\partial_{\bar{z}_{k}} \alpha_{j}\right|_{P, P^{\prime}}$ and

$$
\begin{gathered}
n\{(\underbrace{0}_{q} \mid \underbrace{*}_{n-q})=\partial_{\bar{u}} \widehat{\alpha}=\partial_{z} \alpha \underbrace{\partial_{\bar{u}} z}_{=(0 \mid *)}+\partial_{\bar{z}} \alpha \underbrace{\partial_{\bar{u}} z}_{\text {invertible }} \\
\Rightarrow \partial_{\bar{z}} \alpha=(0 \mid *)\left(\partial_{\bar{u}} \bar{z}\right)^{-1}
\end{gathered}
$$

which is a matrix of rank $\leq n-q$. 
Examples $(n=2$ and $q=1)$. 1. $\varphi=\left|z_{1}\right|^{2}-\left|z_{2}\right|^{2},\left(x, P^{\prime}\right) \stackrel{\mid \Lambda}{\mapsto}\left(x+z_{1}^{\prime}, z_{2}^{\prime}, w^{\prime}+\right.$ $\left.2 i x \bar{z}_{1}^{\prime}\right), \quad \mid \Lambda_{P^{\prime}}^{*}(y)=\left(z_{1}^{\prime}, y, s^{\prime}-2 \operatorname{Im}\left\{z_{2}^{\prime} \bar{y}\right\}+i\left|z_{1}^{\prime}\right|^{2}-i|y|^{2}\right)$. In this case $\mathscr{Z}$ is the identity on $\Omega \times \Sigma$ and $H\left(z, P^{\prime}\right)=2 i \bar{z}_{1}^{\prime}\left(z_{1}-z_{1}^{\prime}\right)-2 i \bar{z}_{2}\left(z_{2}-z_{2}^{\prime}\right)$. The inequality $(2.8)$ reads as

$$
-\left|z_{1}^{\prime}\right|^{2}-\left|z_{2}\right|^{2}+2 \operatorname{Re}\left\{z_{1} \bar{z}_{1}^{\prime}\right\}-\left|z_{2}-z_{2}^{\prime}\right|^{2}\left\langle\left|z_{1}\right|^{2}-\left|z_{2}\right|^{2}-\left|z_{2}-z_{2}^{\prime}\right|^{2}\right.
$$

2. Let us also look at a simple nonrigid example. If $\Sigma$ is given by $\varphi=$ $\left|z_{1}+s\right|^{2}$, then the map

$$
/ \mathbf{\Lambda}: \mathbf{C}_{x} \times \Sigma \rightarrow \mathbf{C}^{3} ; \quad\left(x, P^{\prime}\right) \mapsto\left(z_{1}^{\prime}+x, z_{2}^{\prime}, w^{\prime}-\frac{\bar{z}_{1}^{\prime}+s^{\prime}}{R+i / 2} x\right)
$$

with $R:=\operatorname{Re}\left(\bar{z}_{1}^{\prime}+s^{\prime}\right)$ defines holomorphic curves below the surface. One again has $z\left(u, P^{\prime}\right)=u$ and writing $I:=\operatorname{Im}\left(\bar{z}_{1}^{\prime}+s^{\prime}\right)$ we can put

$$
\begin{aligned}
H\left(z, P^{\prime}\right) & =-\frac{\bar{z}_{1}^{\prime}+s^{\prime}}{R+i / 2}\left(z_{1}-z_{1}^{\prime}\right)-i\left|z_{2}-z_{2}^{\prime}\right|^{2} \\
& =\frac{2(R+i I)(i-2 R)}{1+4 R^{2}}\left(z_{1}-z_{1}^{\prime}\right)-i\left|z_{2}-z_{2}^{\prime}\right|^{2} .
\end{aligned}
$$

It then follows

$$
\begin{aligned}
\operatorname{Im} H & -2 R \operatorname{Re} H=2 R \operatorname{Re}\left(z_{1}-z_{1}^{\prime}\right)-2 I \operatorname{Im}\left(z_{1}-z_{1}^{\prime}\right)-\left|z_{2}-z_{2}^{\prime}\right|^{2} \\
& \Rightarrow \operatorname{Im} H=-2\left|z_{1}^{\prime}-s^{\prime}\right|^{2}+2 \operatorname{Re}\left\{\left(\bar{z}_{1}^{\prime}+s^{\prime}\right)\left(z_{1}+s^{\prime}+\operatorname{Re} H\right)\right\}-\left|z_{2}-z_{2}^{\prime}\right|^{2} \\
& \Rightarrow\left|z_{1}^{\prime}+s^{\prime}\right|^{2}+\operatorname{Im} H \leq\left|z_{1}+s^{\prime}+\operatorname{Re} H\right|^{2}-\frac{1}{2}\left|z_{2}-z_{2}^{\prime}\right|^{2},
\end{aligned}
$$

the latter being nothing else but the convexity inequality (2.8).

Lemma 2.9 gives in this case the smooth maps

$$
\begin{aligned}
& \alpha\left(z, P^{\prime}\right)=\left(\frac{2\left(\bar{z}_{1}^{\prime}+s^{\prime}\right)}{1-2 i R},-\left(\bar{z}_{2}-\bar{z}_{2}^{\prime}\right)\right) \quad \text { and } \\
& \mu\left(P, P^{\prime}\right)=1-2 i \operatorname{Re}\left\{\bar{z}_{1}+s\right\}+i\left(s-s^{\prime}-\operatorname{Re} H\right) .
\end{aligned}
$$

Third step: Application of the homotopy formula. We had seen that we could assume $f \in C^{1}\left(\Sigma, \Lambda^{n+1, n-q+1}\right)$. With the help of Lemma 2.9, we are going to show now that $f$ is in fact cohomologous to a smooth form in the positive part $\{\sigma>0\}$ of the characteristic set.

Set $a: \mathscr{U} \times \mathscr{U} \times \mathbf{R} \rightarrow \mathbf{C}_{n} ;\left(z, s, z^{\prime}, s^{\prime}, \sigma\right) \mapsto \sigma \alpha\left(z, z^{\prime}, s^{\prime}\right)$. By Theorem 1.4 we have the formula

$$
\begin{aligned}
\mathscr{I}^{\varphi} f= & \bar{\partial}_{\Sigma}\left((-1)^{n-q+1} \mathscr{K}_{\Theta}^{a, \varphi} f\right)+(-1)^{n-q} \mathscr{K}_{\Theta}^{a, \varphi}\left(\bar{\partial}_{\Sigma} f\right) \\
& +\mathscr{R}_{\theta}^{a, \varphi} f+(-1)^{n-q+1} \mathscr{K}_{\partial O}^{a, \varphi} f .
\end{aligned}
$$

Since $\operatorname{rank}\left(L_{j} a_{i}\right)<n-q+1$ we can apply Theorem 1.5 and see that the error term $\mathscr{R}_{\mathscr{\theta}}^{a, \varphi} f$ is zero.

In the following equation, instead of $\int_{0}^{1} \varphi_{s}\left(z, \tau s+(1-\tau) s^{\prime}\right) d \tau$, let us write the shorthand $\int_{0}^{1} \varphi_{s}$ and define the pseudodifferential operator $\mathbf{I}_{+}^{\varphi}$ by

$$
\mathbf{I}_{+}^{\varphi} f_{J}(z, s):=\int_{\mathscr{G}} \int_{\sigma>0} e^{i \sigma\left(s-s^{\prime}\right)} f_{J}\left(z, s^{\prime}\right) \frac{1+i \varphi_{s}\left(z, s^{\prime}\right)}{1+i \int_{0}^{1} \varphi_{s}} \frac{d \sigma}{2 \pi} d s^{\prime}
$$


and, of course,

$$
\mathbf{I}_{+}^{\varphi} f(z, s):=\sum_{|J|=n-q+1} \mathbf{I}_{+}^{\varphi} f_{J}(z, s) d \bar{z}_{J} \wedge d z \wedge d w .
$$

In [T3], Trèves assumed the existence of maps $\alpha$ and $\mu$ in Lemma 2.9 and proved

Lemma 2.14. As $\varepsilon \downarrow 0$, the integral $\int_{\sigma>0} e^{-\varepsilon \sigma^{2} \mathscr{J} \varphi} f(z, s, \sigma) d \sigma / 2 \pi$ converges to $\mathcal{F}_{+}^{\varphi} f(z, s)$, and the operator $\mathbf{I}_{+}^{\varphi}$ is congruent to the identity (modulo regularizing operators) in the positive part $\{\sigma>0\}$ of the characteristic set of $\bar{\partial}_{\Sigma}$. Moreover, the limit

- $\mathbf{K}_{\mathscr{O}+}^{a, \varphi} f(z, s):=\lim _{\varepsilon \downarrow 0} \int_{\sigma>0} e^{-\varepsilon \sigma^{2}} \mathscr{K}_{\mathscr{Q}}^{a, \varphi} f(z, s, \sigma) d \sigma / 2 \pi$

exists in $C^{0}\left(\Sigma, \Lambda^{n+1, n-q}\right)$.

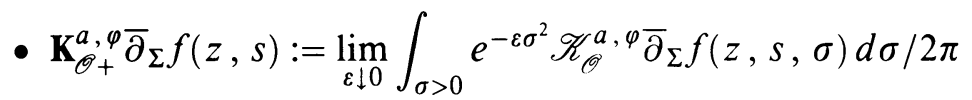

exists in $C_{\varepsilon}^{\infty}\left(\Sigma, \Lambda^{n+1, n-q+1}\right)$.

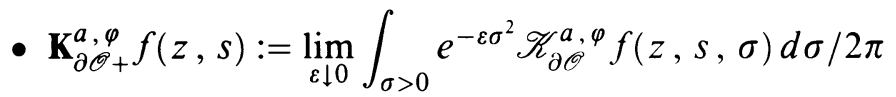

exists in $C^{\infty}\left(\Sigma, \Lambda^{n+1, n-q+1}\right)$.

A proof of this lemma can also be found in [P]. Grosso modo, it goes as follows: In the first integral, we can apply Cauchy's theorem and deform the contour of integration into the line $\left\{\sigma /\left(1+i \int \varphi_{s}\right) \mid \sigma>0\right\}$. Then we obtain,

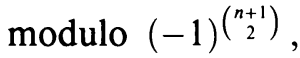

$$
\begin{aligned}
\int_{\sigma>0} & e^{-\varepsilon \sigma^{2}} \mathscr{J}^{\varphi} f_{J}(z, s, \sigma) \frac{d \sigma}{2 \pi} \\
& =\int_{\mathscr{T}} \int_{\sigma} e^{i \sigma\left[s-s^{\prime}+i \varphi(z, s)-i \varphi\left(z, s^{\prime}\right)\right]-\varepsilon \sigma^{2}} f_{J}\left(z, s^{\prime}\right)\left(1+i \varphi_{s}\left(z, s^{\prime}\right)\right) \frac{d \sigma}{2 \pi} d s^{\prime} \\
& =\int_{\mathscr{T}} \int_{\sigma} e^{i \sigma\left(s-s^{\prime}\right)-\varepsilon \sigma^{2} /\left(1+i \int \varphi_{s}\right)^{2}} f_{J}\left(z, s^{\prime}\right) \frac{1+i \varphi_{s}\left(z, s^{\prime}\right)}{1+i \int \varphi_{s}} \frac{d \sigma}{2 \pi} d s^{\prime}
\end{aligned}
$$

which converges to $\mathbf{I}_{+}^{\varphi} f_{J}(z, s)$ as $\varepsilon \downarrow 0$. The symbol of the operator $\mathbf{I}_{+}^{\varphi}$ is

$$
\left.e^{\partial_{\sigma} D_{s^{\prime}}}\left(\frac{1+i \varphi_{s}\left(z, s^{\prime}\right)}{1+i \int \varphi_{s}} H(\sigma)\right)\right|_{s=s^{\prime}} \equiv H(\sigma) \quad\left(\bmod S^{-\infty}(\mathscr{U})\right) .
$$

The operator $\mathbf{I}_{+}^{\varphi}$ is therefore congruent to the identity in the positive part $\{\sigma>0\} \subset T^{*}\left(\mathbf{C}_{z}^{n} \times \mathbf{R}_{s}\right)$ (modulo regularizing operators).

In the next integral, which is of the form

$$
\lim _{\varepsilon \downarrow 0} \int_{\sigma>0} e^{-\varepsilon \sigma^{2}} \int_{\sigma} e^{i \sigma\left[u-w^{\prime}-i \alpha\left(z-z^{\prime}\right)\right]} u \frac{d \sigma}{2 \pi},
$$

one can deform the contour into the line $\{\sigma \mu \mid \sigma>0\}$ and then use the exponential decay coming from the first inequality in Lemma 2.9 in order to show its convergence.

The other two integrals can be handled in a similar way. The additional regularity stems from the assumption that $\bar{\partial}_{\Sigma} f$ is smooth in the first case and 
from the fact that the integration along the boundary $\partial \mathscr{O}$ stays away from the point $(z, s)$ in the second case, because Lemma 2.9 then yields a strict inequality $\operatorname{Im}\{\sigma \mu[\cdots]\} \geq \delta>0$.

Conclusion. The homotopy formula allows us to write (cf. (2.11))

$$
\begin{aligned}
\mathbf{I}_{+}^{\varphi} f(z, s)= & \bar{\partial}_{\Sigma}\left((-1)^{n-q+1} \mathbf{K}_{\mathscr{\mathscr { C }}+}^{a, \varphi} f\right)+(-1)^{n-q} \mathbf{K}_{\mathscr{O}+}^{a, \varphi} \bar{\partial}_{\Sigma} f \\
& +(-1)^{n-q+1} \mathbf{K}_{\partial \mathscr{O}+}^{a, \varphi} f .
\end{aligned}
$$

Thanks to our choice of the map $a$ and using a change of contour induced by $\mu$ we just saw that the currents $\mathbf{K}_{\mathscr{O}+} \bar{\partial}_{\Sigma} f$ and $\mathbf{K}_{\partial \mathscr{O}+} f$ are smooth. Since $\mathbf{I}_{+}^{\varphi} f$ is congruent to the identity (modulo regularizing operators) in the positive part $\{\sigma>0\}$ of the characteristic set, we have $\operatorname{WF}\left(\mathbf{I}_{+}^{\varphi} f-f\right) \cap\{\sigma>0\}=\varnothing$. Letting $u:=(-1)^{n-q+1} \mathbf{K}_{\mathscr{O}+}^{a, \varphi} f$ we have therefore shown that

$$
\begin{gathered}
\mathrm{WF}\left(f-\bar{\partial}_{\Sigma} u\right) \cap\{\sigma>0\}=\mathrm{WF}\left(\mathbf{I}_{+}^{\varphi} f-\bar{\partial}_{\Sigma} u\right) \cap\{\sigma>0\}=\varnothing \\
\Rightarrow \mathrm{WF}\left(f-\bar{\partial}_{\Sigma} u\right) \subset\{\sigma<0\} .
\end{gathered}
$$

Thus we have proven the last of four claims made in Theorem 2.7, namely the implication $\Sigma q$-holoconcave up $\Rightarrow \forall q^{\prime}<q \bar{\partial}_{\Sigma}\left(n-q^{\prime}\right)$-hypoelliptic ${ }^{+}$. We finish this section by indicating the modifications necessary to obtain the other three claims.

Under the same hypothesis, $\Sigma q$-holoconcave up, we could have considered a form $f \in C^{1}\left(\Sigma, \Lambda^{n+1, q-1}\right)$. However, in (2.11) the application of Theorem 1.5 became impossible unless we changed the construction of our Lemma 2.9. If, in the second step, we hand changed the roles played by $(z, s)$ and $\left(z^{\prime}, s^{\prime}\right)$, we would have gotten the inequalities $\operatorname{Im}\{\mu[\cdots]\} \leq 0$ and $\operatorname{rank}\left(L_{j}^{\prime} \alpha_{i}\right) \leq n-q$ in Lemma 2.9. Hence we could get rid of the error term in (2.11). In the third step, we would need to integrate on the domain $\{\sigma<0\}$ and define $\mathbf{I}_{-}^{\varphi} f$, $\mathbf{K}_{\mathscr{O}_{-}}, \mathbf{K}_{\partial \mathscr{O}_{-}}$. In conclusion we obtain $\operatorname{WF}\left(f-\bar{\partial}_{\Sigma} u\right) \subset\{\sigma>0\}$, i.e. $\bar{\partial}_{\Sigma}$ is $(q-1)$-hypoelliptic ${ }^{-}$.

Under the hypothesis of $q$-holoconcavity down however, the inequality (2.8) changes the direction, provided we add rather than subtract the quadratic term $\frac{1}{2}\left|u_{(2)}-z_{(2)}^{\prime}\right|^{2}$. Starting with a current $f \in \mathscr{D}^{\prime}\left(\Sigma, \Lambda^{n+1, n-q+1}\right)$ we have the inequalities $\operatorname{Im}\{\mu[\cdots]\} \leqslant 0$ and $\operatorname{rank}\left(L_{j} \alpha_{i}\right)<n-q+1$ in Lemma 2.9. In order to conclude that $\bar{\partial}_{\Sigma}$ is $(n-q+1)$-hypoelliptic ${ }^{-}$, we then integrate on $\{\sigma<0\}$.

If $\Sigma$ is $q$-holoconcave down and $f \in \mathscr{D}^{\prime}\left(\Sigma, \Lambda^{n+1, q-1}\right)$ we must change the direction of the inequality (2.8) as well as exchange the roles played by $(z, s)$ and $\left(z^{\prime}, s^{\prime}\right)$ respectively, in the second step of the proof. Lemma 2.9 then yields $\operatorname{Im}\{\mu[\cdots]\}>0$ and $\operatorname{rank}\left(L_{j}^{\prime} \alpha_{i}\right) \leq n-q$, which leads to the conclusion that $\bar{\partial}_{\Sigma}$ is $(q-1)$-hypoelliptic ${ }^{+}$.

\section{THE GENERAL CASE}

3.1. Definitions. We go back now to the general setting of the preliminary section 1.1. $\mathscr{M}$ is again a differentiable manifold of dimension $m+n$ carrying a CR-structure, and we consider its fine embedding $\Sigma=p(\mathscr{M}) \subset \mathbf{C}^{m}$, of codimension $d=m-n$ and tangent to the plane $\mathbf{C}_{z}^{n} \times \mathbf{R}_{s}^{d}$ at the origin.

For convenience, we recall 
Definition 3.1. Let $q \in \mathbf{Z}$. We call the operator $\bar{\partial}_{\Sigma} q$-hypoelliptic if every germ of current $v \in \mathscr{D}^{\prime}\left(\Sigma, \Lambda^{m, q}\right)$ with $\bar{\partial}_{\Sigma} v \in C^{\infty}\left(\Sigma, \Lambda^{m, q+1}\right)$ is cohomologous to the germ of a smooth form.

We again seek a sufficient condition for $q$-hypoellipticity, for $0 \leq q \leq n$. But we are not going to microlocalize the problem as we did before in the hypersurface case. This time the variable $\sigma$ shall vary in the whole fiber of the characteristic set.

Let $S_{d-1} \subset \mathbf{R}_{d} \subset \mathbf{C}_{d}$ be the unit cosphere.

Definition 3.2. $\Sigma$ is called $q$-holoconvex, if there exist a complex cone containing $S_{d-1}, \mathscr{C}=\left\{\sigma \in \mathbf{C}_{d} \backslash 0|| \operatorname{Im} \sigma|<\kappa| \operatorname{Re} \sigma \mid, 0<\kappa<1\right\}$, a holomorphic map $\mathscr{P}: \mathscr{C} \rightarrow M_{n}(\mathbf{C})$, positive homogeneous of degree zero, and a smooth map / $\mathbf{\Lambda}: \mathbf{C}_{u}^{n} \times \Sigma \times \mathscr{C} \rightarrow \mathbf{C}^{m} ;\left(0, P^{\prime}, \sigma\right) \mapsto P^{\prime}$, also holomorphic and positive homogeneous of degree zero in $\sigma$, such that the following four conditions are satisfied:

$$
\forall_{\sigma \in S_{d-1}} \mathscr{P}(\sigma) \text { is a projection of rank } q .
$$

$$
\forall_{\sigma} \forall_{P^{\prime}} \quad \text { the map } u \mapsto / \Lambda\left(\mathscr{P} u, P^{\prime}, \sigma\right) \text { is holomorphic. }
$$

$$
\begin{gathered}
\left.\forall_{\sigma \in S_{d-1}} \partial_{u} \Lambda\right|_{0,0, \sigma} \in \mathrm{Gl}_{n}(\mathbf{C}) . \\
\forall_{u} \forall_{P^{\prime}} \forall_{\sigma} \quad \sigma\left[\varphi\left(\Lambda, \operatorname{Re} / \Lambda^{n+1, \ldots, m}\right)-\operatorname{Im} /\left.\Lambda^{n+1, \ldots, m}\right|_{\mathscr{P}(\sigma) u, P^{\prime}, \sigma}\right] \geq 0
\end{gathered}
$$

NB: As before, we put $\Lambda=/ \Lambda^{1, \ldots, n}$. Inequality (3.4) is meant to be strict unless $\mathscr{P}(\sigma) u=0$. We also remark that this definition is compatible with Definition 2.5, i.e., in codimension $d=1 \Sigma$ is $q$-holoconvex iff it is both holoconcave up and holoconcave down.

Example $(n=d=2) . \Sigma=\left\{(z, w) \in \mathbf{C}^{4} \mid \operatorname{Im} w=\varphi(z)=\left(\left|z_{1}\right|^{2}-\left|z_{2}\right|^{2}\right.\right.$, $\left.\left.w \operatorname{Im}\left\{\bar{z}_{1} z_{2}\right\}\right)\right\}$ is 1-holoconvex: for $\sigma \in S_{1}, P^{\prime}=\left(z^{\prime}, w^{\prime}\right) \in \Sigma$, let $\mathscr{P}(\sigma)=$ $\left(\begin{array}{ll}1 & 0 \\ 0 & 0\end{array}\right)$ be the projection on the first component and let $\Lambda\left(u, P^{\prime}, \sigma\right)$ be defined by the equations

$$
\Lambda\left(u, P^{\prime}, \sigma\right)=\left(z^{\prime}+u_{1}\left(\begin{array}{c}
1+\sigma_{1}-i \sigma_{2} \\
1-\sigma_{1}+i \sigma_{2}
\end{array}\right)+u_{2}\left(\begin{array}{c}
-1+\sigma_{1}+i \sigma_{2} \\
1+\sigma_{1}+i \sigma_{2}
\end{array}\right)\right)
$$

and

$$
\mathbf{\Lambda}^{3,4}\left(u, P^{\prime}, \sigma\right)=\left(w^{\prime}+2 i u_{1}\left(\left.\partial_{x} \varphi\right|_{P^{\prime}}\right)\left(\begin{array}{c}
1+\sigma_{1}-i \sigma_{2} \\
1-\sigma_{1}+i \sigma_{2}
\end{array}\right)\right) .
$$

Then $\Lambda$ is holomorphic in $u_{1}, \sigma$ and

$$
\left.\partial_{u} \Lambda\right|_{0,0, \sigma}=\left(\begin{array}{cc}
1+\sigma_{1}-i \sigma_{2} & -1+\sigma_{1}+i \sigma_{2} \\
1-\sigma_{1}+i \sigma_{2} & 1+\sigma_{1}+i \sigma_{2}
\end{array}\right) \in \mathrm{Gl}_{2}(\mathbf{C}) .
$$


Moreover, we calculate

$$
\begin{aligned}
\varphi(\Lambda, & \left.\operatorname{Re} / \Lambda^{3,4}\right)-\operatorname{Im} / \Lambda^{3,4}\left(\left(u_{1}, 0\right), P^{\prime}, \sigma\right) \\
= & \varphi\left(z^{\prime}+u_{1}\left(\begin{array}{c}
1+\sigma_{1}-i \sigma_{2} \\
1-\sigma_{1}+i \sigma_{2}
\end{array}\right)\right) \\
& \quad-\operatorname{Im}\left\{w^{\prime}+2 i u_{1}\left(\begin{array}{cc}
\bar{z}_{1}^{\prime} & -\bar{z}_{2}^{\prime} \\
i \bar{z}_{2}^{\prime} & -i \bar{z}_{1}^{\prime}
\end{array}\right)\left(\begin{array}{c}
1+\sigma_{1}-i \sigma_{2} \\
1-\sigma_{1}+i \sigma_{2}
\end{array}\right)\right\} \\
= & \left|u_{1}\right|^{2}\left(\begin{array}{c}
\left|1+\sigma_{1}-i \sigma_{2}\right|^{2}-\left|1-\sigma_{1}+i \sigma_{2}\right|^{2} \\
2 \operatorname{Im}\left\{\left(1+\sigma_{1}+i \sigma_{2}\right)\left(1-\sigma_{1}+i \sigma_{2}\right)\right\}
\end{array}\right) \\
= & 4\left|u_{1}\right|^{2}\left(\begin{array}{c}
\sigma_{1} \\
\sigma_{2}
\end{array}\right) .
\end{aligned}
$$

This example can be generalized as follows.

Proposition 3.3. Suppose $\Sigma=\left\{(z, w) \in \mathbf{C}^{n+d} \mid \operatorname{Im} w=\varphi(z, \operatorname{Re} w)\right\}$ and for all $\sigma \in S_{d-1}$ the matrix $\left.\partial \bar{\partial}(\sigma \cdot \varphi)\right|_{0}=\sum_{k=1}^{d} \sigma_{k}\left(\varphi \frac{k}{z_{i} z_{j}}\right)_{1 \leq i, j \leq n}$ has exactly $q$ positive eigenvalues $0<\lambda_{1}(\sigma) \leq \cdots \leq \lambda_{q}(\sigma)$. Then $\Sigma$ is q-holoconvex.

Let us remark that in the preceding example we had $\lambda_{1}(\sigma)=1$ for all $\sigma$, because the matrix

$$
\left.\partial \bar{\partial}(\sigma \cdot \varphi)\right|_{0}=\sigma_{1}\left(\begin{array}{cc}
1 & 0 \\
0 & -1
\end{array}\right)+\sigma_{2}\left(\begin{array}{cc}
0 & -i \\
i & 0
\end{array}\right)
$$

had eigenvalues 1 and -1 for any $\sigma \in S_{1}$.

Proof of the proposition. After a holomorphic coordinate change in the $w$ variables, we can assume that, for all $1 \leq l \leq d, \varphi_{l}$ is a hermitian quadratic form modulo $O\left(|z|^{3}+|s|^{3}\right)$. In fact, since $w=s+i \varphi(z, s), \varphi$ real, $\varphi(0)=0$, and $d \varphi(0)=0$ we can take

$$
\tilde{w}_{l}:=w_{l}-\sum_{j=1}^{n} \sum_{k=1}^{d} a_{j k}^{(l)} z_{j} w_{k}-\left.\frac{i}{2} \sum_{j, k=1}^{n} \partial_{z_{j} z_{k}} \varphi\right|_{0} z_{j} z_{k}-\sum_{j, k=1}^{d} b_{j k}^{(l)} w_{j} w_{k}
$$

Provided the coefficients $a_{j k}^{(l)}, b_{j k}^{(1)} \in \mathbf{C}$ are well chosen we obtain

$$
\begin{aligned}
\tilde{s} & =\operatorname{Re} \tilde{w}=s+O\left(|z|^{2}+|s|^{2}\right), \\
\tilde{\varphi}(z, \tilde{s}) & =\operatorname{Im} \tilde{w}=\sum_{1 \leq j, k \leq n} c_{j k} z_{j} \bar{z}_{k}+O\left(|z|^{3}+|\tilde{s}|^{3}\right), \quad c_{j k}=\bar{c}_{k j} .
\end{aligned}
$$

The next thing to do is to choose a circle in the complex plane containing all the values $\lambda_{j}(\sigma)$, for $j=1, \ldots, q, \sigma \in S_{d-1}$ and not touching the imaginary axis, and to put

$$
\mathscr{P}(\sigma):=\frac{1}{2 i \pi} \oint \frac{d z}{z I_{n}-\left.\partial \bar{\partial}(\sigma \cdot \varphi)\right|_{0}} .
$$

Let $V_{\lambda_{j}(\sigma)} \subset \mathbf{C}^{n}$ be the eigenspace of the matrix $\left.\partial \bar{\partial}(\sigma \cdot \varphi)\right|_{0}$ associated to the eigenvalue $\lambda_{j}(\sigma)$. Then $\left.\partial \bar{\partial}(\sigma \cdot \varphi)\right|_{0}$ is positive definite on $V_{\sigma}^{+}:=\bigoplus_{j=1}^{q} V_{\lambda_{j}(\sigma)}$. We have $\operatorname{dim}_{\mathbf{C}} V_{\sigma}^{+}=q . \mathscr{P}(\sigma)$ is the orthogonal projection of $\mathbf{C}^{n}$ onto $V_{\sigma}^{+}$.

Now let $u \in \mathbf{C}^{n}, P^{\prime} \in \Sigma, \sigma \in S_{d-1}$. We put

$$
\left.D_{z} \varphi\right|_{P^{\prime}}:=\left(1-\left.i \varphi_{s}\right|_{z^{\prime}, s^{\prime}}\right)^{-1}\left(\left.\partial_{z} \varphi\right|_{z^{\prime}, s^{\prime}}\right) \in M_{d, n}(\mathbf{C})
$$


and define

$$
/ \mathbf{\Lambda}\left(u, P^{\prime}, \sigma\right):=\left(z^{\prime}+u, w^{\prime}+\left.2 i D_{z} \varphi\right|_{P^{\prime}} u\right)
$$

For all $\sigma,\left.\partial_{u} \Lambda\right|_{0,0, \sigma}$ is the identity matrix. In order to show that $\Lambda$ satisfies the property (3.4) we need to estimate the number

$$
\begin{gathered}
\sigma\left[\varphi\left(\Lambda, \operatorname{Re} / \Lambda^{n+1, \ldots, m}\right)-\operatorname{Im} / \Lambda^{n+1, \ldots, m}\left(\mathscr{P}(\sigma) u, P^{\prime}, \sigma\right)\right] \\
=\sigma\left[\varphi \left(z^{\prime}+\mathscr{P} u,\right.\right. \\
\left.s^{\prime}-2 \operatorname{Im}\left\{\left.D_{x} \varphi\right|_{P^{\prime}} \mathscr{P} u\right\}\right) \\
\left.-\varphi\left(z^{\prime}, s^{\prime}\right)-2 \operatorname{Re}\left\{\left.D_{z} \varphi\right|_{P^{\prime}} \mathscr{P} u\right\}\right] .
\end{gathered}
$$

The term in the brackets vanishes to first order and we choose coordinates so that the quadratic term in the development of $\varphi$ has no harmonic term. It follows that our previous expression is

$$
\cdots=\sigma\left[\left.u^{*} \mathscr{P}^{*}(\sigma) \partial \bar{\partial} \varphi\right|_{0} \mathscr{P}(\sigma) u+O\left(|\mathscr{P}(\sigma) u|^{2}\left(|\mathscr{P}(\sigma) u|+\left|z^{\prime}\right|+\left|s^{\prime}\right|\right)\right)\right]
$$

which is positive for small $z^{\prime}, s^{\prime}$ and small $\mathscr{P}(\sigma) u \neq 0$, since $\left.\partial \bar{\partial}(\sigma \cdot \varphi)\right|_{0}$ is a positive definite quadratic form on the image of $\mathscr{P}(\sigma)$.

\subsection{The theorem.}

Theorem 3.4. Let $\Sigma$ be a submanifold of arbitrary codimension in $\mathbf{C}^{m}$. If $\Sigma$ is $q$-holoconvex then the operator $\bar{\partial}_{\Sigma}$ is $q^{\prime}$-hypoelliptic and $\left(n-q^{\prime}\right)$-hypoelliptic for all $q^{\prime}<q$.

Example $(n=d=4)$. Let $a, c \in \mathbf{R}$ and $b, d \in \mathbf{C}$ be nonzero numbers. Then the system

$$
\begin{aligned}
& L_{1}=\partial_{\bar{z}_{1}}-i a z_{1} \partial_{s_{1}}-b z_{2} \partial_{s_{2}}, \\
& L_{2}=\partial_{\bar{z}_{2}}+i a z_{2} \partial_{s_{1}}+\bar{b} z_{1} \partial_{s_{2}}, \\
& L_{3}=\partial_{\bar{z}_{3}}-i c z_{3} \partial_{s_{3}}-d z_{3} \partial_{s_{4}}, \\
& L_{4}=\partial_{\bar{z}_{4}}+i c z_{4} \partial_{s_{3}}+\bar{d} z_{3} \partial_{s_{4}}
\end{aligned}
$$

is $q$-hypoelliptic for $q=0,1,3,4$. In fact, by Proposition 3.3, the manifold $\Sigma$ defined by $\varphi(z)=\left(a\left|z_{1}\right|^{2}-a\left|z_{2}\right|^{2}, 2 \operatorname{Im} b \bar{z}_{1} z_{2}, c\left|z_{3}\right|^{2}-c\left|z_{4}\right|^{2}, 2 \operatorname{Im} d \bar{z}_{3} z_{4}\right)$ is 2 -holoconvex.

Corollary 3.5. Suppose $\Sigma$ is a 1-holoconvex submanifold of arbitrary codimension in $\mathbf{C}^{m}$, and $u$ is a distribution on $\Sigma$. Then $u$ is smooth if and only if $\bar{\partial}_{\Sigma} u$ is smooth.

Example $(n=d=2)$. Assume $u(z, s)$ is a distribution on $\mathbf{C}^{2} \times \mathbf{R}^{2}$ such that

$$
\partial_{\bar{z}_{1}} u-2 i z_{1}\left|z_{1}\right|^{2} \partial_{s_{1}} u-z_{2} \partial_{s_{2}} u
$$

and

$$
\partial_{\bar{z}_{2}} u+2 i z_{2}\left|z_{2}\right|^{2} \partial_{s_{1}} u+z_{1} \partial_{s_{2}} u
$$

are both smooth. Then the corollary says that $u$ is a smooth function.

In fact, the two vector fields define the tangential Cauchy-Riemann operator on the manifold

$$
\Sigma=\left\{(z, w) \in \mathbf{C}^{4} \mid \operatorname{Im} w=\varphi(z)=\left(\left|z_{1}\right|^{4}-\left|z_{2}\right|^{4}, 2 \operatorname{Im} \bar{z}_{1} z_{2}\right)\right\}
$$

which is 1-holoconvex: take the projection

$$
\mathscr{P}(\sigma):=\left(\begin{array}{cc}
1+\sigma_{1}-i \sigma_{2} & 0 \\
1-\sigma_{1}+i \sigma_{2} & 0
\end{array}\right)
$$


and define

$$
\mathbf{\Lambda}\left(u, P^{\prime}, \sigma\right):=\left(z^{\prime}+u, w^{\prime}+2 i\left(\begin{array}{cc}
2 \bar{z}_{1}^{\prime}\left|z_{1}^{\prime}\right|^{2} & -2 \bar{z}_{2}^{\prime}\left|z_{2}^{\prime}\right|^{2} \\
i \bar{z}_{2}^{\prime} & -i \bar{z}_{1}^{\prime}
\end{array}\right) u\right)
$$

The conditions (3.1)-(3.4) are satisfied: $\left.\partial_{u} \Lambda\right|_{0,0, \sigma}$ is the identity matrix for all $\sigma \in S_{1}$ and letting $\left(\begin{array}{l}x_{1} \\ x_{2}\end{array}\right):=\mathscr{P} u$ and $\bar{z}_{j}^{\prime} x_{j}=\left|\bar{z}_{j}{ }_{j}^{\prime} x_{j}\right| e^{i \theta_{j}}$ we have

$$
\begin{aligned}
& \varphi(\Lambda,\left.\operatorname{Re} / \Lambda^{3,4}\right)-\operatorname{Im} / \Lambda^{3,4}\left(\mathscr{P} u, P^{\prime}, \sigma\right) \\
&=\left(\begin{array}{c}
\left.\left|2 z_{1}^{\prime} x_{1}+x_{1}^{2}\right|^{2}+2 \operatorname{Re}\left\{\bar{z}_{1}^{\prime 2} x_{1}^{2}\right\}-\left|2 z_{2}^{\prime} x_{2}+x_{2}^{2}\right|^{2}-2 \operatorname{Re}\left\{\bar{z}_{2}^{\prime 2} x_{2}^{2}\right\}\right) \\
2 \operatorname{Im}\left\{\bar{x}_{1} x_{2}\right\}
\end{array}\right) \\
& \quad=\left|u_{1}\right|^{2}\left(\begin{array}{c}
\left(2+2 \sigma_{1}\right) a-\left(2-2 \sigma_{1}\right) b \\
4 \sigma_{2}
\end{array}\right)
\end{aligned}
$$

with $a=2\left|z_{1}^{\prime}\right|^{2}+\left(\left|x_{1}\right|+2\left|z_{1}^{\prime}\right| \cos \theta_{1}\right)^{2}$ and $b=2\left|z_{2}^{\prime}\right|^{2}+\left(\left|x_{2}\right|+2\left|z_{2}^{\prime}\right| \cos \theta_{2}\right)^{2}$ nonnegative and small. Then it follows that

$$
\begin{aligned}
& \sigma\left[\varphi\left(z^{\prime}+\mathscr{P} u\right)-\operatorname{Im} / \Lambda^{3,4}\left(\mathscr{P} u, P^{\prime}, \sigma\right)\right] \\
& \quad=4\left|u_{1}\right|^{2}\left((a+b) \sigma_{1}^{2}+\frac{a-b}{2} \sigma_{1}+1-\sigma_{1}^{2}\right)>0
\end{aligned}
$$

with equality only for $u_{1}=0$ or $a=b=1-\sigma_{1}^{2}=0$, that is, for $\mathscr{P} u=0$.

3.3. Proof. We assume $\Sigma=\left\{(z, w) \in \mathbf{C}^{n} \times \mathbf{C}^{d} \mid \operatorname{Im} w \in \varphi(z, \operatorname{Re} w), z \in \Omega\right.$, $\operatorname{Re} w \in \mathscr{T}\}$ is $q$-holoconvex and $f$ is a current of bidegree $(m, n-q+1)$ such that $\bar{\partial}_{\Sigma} f$ is smooth. Exactly as in the first step of $\S 2.5$, general distribution theory allows us to assume without restriction that

$$
f=\sum_{|J|=n-q+1} f_{J}(z, s) d \bar{z}_{J} \wedge d z \wedge d w \in C^{1}\left(\Sigma, \Lambda^{m, n-q+1}\right) .
$$

We show now that $f$ is cohomologous to a smooth form.

Lemma 3.6. There are smooth maps $\alpha: \mathscr{U}_{z, s} \times \mathscr{U}_{z^{\prime}, s^{\prime}} \times \mathscr{C}_{\sigma} \rightarrow M_{d, n}(\mathbf{C})$ and $\mu: \mathscr{U} \times \mathscr{U} \times \mathscr{C} \rightarrow M_{d}(\mathbf{C})$ such that for all points $P, P^{\prime} \in \Sigma$ and for all $\sigma \in \mathscr{C}$ one has $\sigma$ real $\Rightarrow \operatorname{Re} \mu=I_{d}$ and

$$
\begin{gathered}
\sigma \text { real } \Rightarrow \operatorname{Im}\left\{\sigma \mu\left[w-w^{\prime}-i \alpha\left(z-z^{\prime}\right)\right]\right\}>0, \\
\operatorname{rank}\left(L_{j}(\sigma \alpha)_{i}\right)_{1 \leq i, j \leq n} \leq n-q .
\end{gathered}
$$

Moreover, $\alpha$ and $\mu$ can be chosen holomorphic and positive homogeneous of degree zero in $\sigma$.

Proof. According to Definition 3.2, we have at our disposal projections $\mathscr{P}(\sigma)$ and a map $/ \boldsymbol{\Lambda}=\left(\Lambda, / \Lambda^{n+1}, \ldots, m\right)$. Let us put $\mathscr{Q}=I_{n}-\mathscr{P}$. For all $\sigma \in S_{d-1}$ we have $\mathbf{C}^{n}=$ Image $\mathscr{P}(\sigma) \oplus$ Image $\mathscr{Q}(\sigma)$. Momentarily, we introduce two more abbreviations, namely

$$
\left.\Lambda\right|_{\sigma}:=\left.\partial_{u} \Lambda\right|_{0,0, \sigma} \quad \text { and } \quad \phi\left(u, P^{\prime}, \sigma\right):=\varphi\left(z^{\prime}+\left.\Lambda\right|_{\sigma} \mathscr{Q}\left(u-z^{\prime}\right), s^{\prime}\right) .
$$

Here we assume again, as always, that $P^{\prime}=\left(z^{\prime}, w^{\prime}\right)=\left(z^{\prime}, s^{\prime}+i \varphi\left(z^{\prime}, s^{\prime}\right)\right) \in$ $\Sigma$. $\phi$ is invariant under the projection $\mathscr{Q}$, that is, $\forall_{u, P^{\prime}, \sigma} \phi\left(\mathscr{Q} u, P^{\prime}, \sigma\right)=$ $\phi\left(u, P^{\prime}, \sigma\right)$. 
We copy as closely as possible the construction in the proof of Lemma 2.9 and define manifolds $/ \mathbf{\Lambda}^{*} \subset \Sigma$ transversal to $/ \boldsymbol{\Lambda}$ as follows. Let

$$
\begin{aligned}
s^{*}\left(u, P^{\prime}, \sigma\right):= & s^{\prime}-2 \operatorname{Re}\left\{\left.\left(1+i \varphi_{s}\right)^{-1} i \phi_{\bar{u}}\right|_{z^{\prime}, P^{\prime}, \sigma}\left(\bar{u}-\bar{z}^{\prime}\right)\right\} \\
& +2 \operatorname{Re}\left(\begin{array}{c}
{ }^{t}\left(\bar{u}-\bar{z}^{\prime}\right)^{t} \overline{\mathscr{Q}} H^{(1)} \overline{\mathscr{Q}}\left(\bar{u}-\bar{z}^{\prime}\right) \\
\vdots \\
{ }^{t}\left(\bar{u}-\bar{z}^{\prime}\right)^{t} \overline{\mathscr{Q}} H^{(d)} \overline{\mathscr{Q}}\left(\bar{u}-\bar{z}^{\prime}\right)
\end{array}\right),
\end{aligned}
$$

where the matrices $H^{(l)}\left(P^{\prime}, \sigma\right) \in M_{n}(\mathbf{C}), 1 \leq l \leq d$, will be defined later. Then we define

$$
\Sigma \ni / \Lambda^{*}\left(u, P^{\prime}, \sigma\right):=\left(z^{\prime}+\left.\Lambda\right|_{\sigma} \mathscr{Q}\left(u-z^{\prime}\right), s^{*}+i \varphi\left(z^{\prime}+\left.\Lambda\right|_{\sigma} \mathscr{Q}\left(u-z^{\prime}\right), s^{*}\right)\right) \text {. }
$$

Note that $s^{*}$ and $/ \Lambda^{*}$ are independent of $\mathscr{P} u$. In the preceding section there was no dependence of $\sigma$, and $\left.\Lambda\right|_{\sigma} \mathscr{Q}\left(u-z^{\prime}\right)$ was simply equal to $u_{(2)}-z_{(2)}^{\prime}$. There, it was absolutely no problem to pick a direction transversal to all supporting manifolds $/ \boldsymbol{\Lambda}_{P^{\prime}}$. This is the reason why, in the hypersurface case, we only needed to define $/ \Lambda\left(\left(u_{(1)}, 0\right), P^{\prime}\right)$, not $/ \Lambda\left(u, P^{\prime}\right)$. In our present circumstances however, this would be insufficient, because for topological reasons it is in general not possible to define the transversal directions of $\left.\partial_{u_{(1)}} \Lambda\right|_{0,0, \sigma} \in M_{n, q}(\mathbf{C})$ depending continuously on $\sigma \in S_{d-1}$.

We observe that $/ \Lambda^{*}\left(z^{\prime}, P^{\prime}, \sigma\right)=P^{\prime}$.

Definition 3.7. Let $z\left(u, P^{\prime}, \sigma\right):=\Lambda\left(\mathscr{P}\left(u-z^{\prime}\right), / \Lambda^{*}\left(u, P^{\prime}, \sigma\right), \sigma\right)$. We introduce a smooth map $\mathscr{Z}$ as follows: $\mathscr{Z}: \mathbf{C}^{n} \times \Sigma \times \mathscr{C} \rightarrow \mathbf{C}^{n} \times \Sigma \times \mathscr{C} ;\left(u, P^{\prime}, \sigma\right) \mapsto$ $\left(z\left(u, P^{\prime}, \sigma\right), P^{\prime}, \sigma\right)$.

Immediately we should remark that

$$
\forall u, P^{\prime}, \sigma \quad z\left(z^{\prime}+\mathscr{Q}\left(u-z^{\prime}\right), P^{\prime}, \sigma\right)=\Lambda^{*}\left(u, P^{\prime}, \sigma\right)=z^{\prime}+\left.\Lambda\right|_{\sigma} \mathscr{Q}\left(u-z^{\prime}\right) .
$$

We now apply the chainrule and (3.2) to calculate the complex Jacobian matrix $\left.\partial_{u} z\right|_{z^{\prime}, P^{\prime}, \sigma}=\left.\partial_{u} \Lambda\right|_{0, P^{\prime}, \sigma} \cdot \mathscr{P}+\left.\left.\Lambda\right|_{\sigma} \cdot \mathscr{Q} \approx \partial_{u} \Lambda\right|_{0,0, \sigma}$. Thanks to hypothesis (3.3), this proves the first claim of the following remark.

Remark 3.8. For all $P^{\prime} \in \Sigma$ sufficiently close to the origin, and for $\sigma \in S_{d-1}$, $\left.\partial_{u} z\right|_{z^{\prime}, P^{\prime}, \sigma}$ is invertible, $\left.\partial_{\bar{u}} z\right|_{z^{\prime}, P^{\prime}, \sigma}=0$ and $\left.\partial_{\bar{u} \bar{u}}^{2} z\right|_{z^{\prime}, P^{\prime}, \sigma}=0$. Moreover, $z$ is holomorphic on the image of the projection $\mathscr{P}$ (i.e., the map $u \mapsto$ $z\left(\mathscr{P}(\sigma) u, P^{\prime}, \sigma\right)$ is holomorphic for any fixed $\left.P^{\prime}, \sigma\right)$ and positive homogeneous of degree zero in $\sigma$.

Proof.

$$
\begin{aligned}
\left.\partial_{\bar{u}} z\right|_{z^{\prime}, P^{\prime}, \sigma} & =\underbrace{\left.\partial_{\bar{u}} \Lambda\right|_{0, P^{\prime}, \sigma} \cdot \overline{\mathscr{P}}}_{(\stackrel{3.2)}{=} 0}+\underbrace{\left.\partial_{P^{\prime}} \Lambda\right|_{0, P^{\prime}, \sigma}}_{=\left(I_{2 n} \mid 0\right)} \partial_{\bar{u}} /\left.\Lambda^{*}\right|_{z^{\prime}, P^{\prime}, \sigma} \\
& =\left.\partial_{\bar{u}}\left(\left.\Lambda\right|_{\sigma} \mathscr{Q} u\right)\right|_{z^{\prime}, \sigma}=0 .
\end{aligned}
$$

The second antiholomorphic derivatives of $z$ vanish in the directions of the image of the projection $\mathscr{Q}$, because of (3.8). But they are also zero in $\mathscr{P}$ directions, because $/ \Lambda^{*}\left(\mathscr{P} u, P^{\prime}, \sigma\right)=/ \mathbf{\Lambda}^{*}\left(0, P^{\prime}, \sigma\right)$ is independent of $u$ and by (3.2) the map $u \mapsto z\left(\mathscr{P} u, P^{\prime}, \sigma\right)=\Lambda\left(\mathscr{P}\left(u-z^{\prime}\right), \quad \Lambda^{*}\left(\mathscr{P} u, P^{\prime}, \sigma\right), \sigma\right)$ is holomorphic. 
The remark implies that the real Jacobian $\partial_{u}^{\mathbf{R}} z$ is invertible at points $\left(z^{\prime}, P^{\prime}, \sigma\right)$. We can therefore choose the neighbourhoods of zero $\Omega \odot \mathbf{C}^{n}$, $\mathscr{T} \subseteq \mathbf{R}^{s}$, the open set $\widehat{\Omega} \subset \mathbf{C}^{n} \times \Sigma \times \mathscr{C}$ containing $0 \times 0 \times S_{d-1}$ and the number $0<\kappa<1$ small enough so that $\mathscr{Z}: \widehat{\Omega} \rightarrow \Omega \times \Sigma \times \mathscr{C}$ is a diffeomorphism.

Definition 3.9. To each $\left(u, P^{\prime}, \sigma\right) \in \widehat{\Omega}$, we associate a vector in $\mathbf{C}^{d}$,

$$
\begin{aligned}
\widehat{H}\left(u, P^{\prime}, \sigma\right):= & \mid \mathbf{\Lambda}^{n+1, \ldots, m}\left(\mathscr{P}\left(u-z^{\prime}\right), \mid \mathbf{\Lambda}^{*}\left(u, P^{\prime}, \sigma\right), \sigma\right) \\
& -w^{\prime}-i\left|\mathscr{Q}\left(u-z^{\prime}\right)\right|^{2 t} \sigma / \sqrt{\sigma \cdot{ }^{t} \sigma} .
\end{aligned}
$$

This map is smooth and in $\sigma$ it is holomorphic and positive homogeneous of degree zero.

Remark 3.10. For all points $\left(z^{\prime}, P^{\prime}, \sigma\right) \in \widehat{\Omega}$ with $P^{\prime}=\left(z^{\prime}, w^{\prime}\right)$ we have $\widehat{H}\left(z^{\prime}, P^{\prime}, \sigma\right)=0,\left.\partial_{\bar{u}} \widehat{H}\right|_{z^{\prime}, P^{\prime}, \sigma}=0$ and $\left.\partial_{\bar{u} u}^{2} \widehat{H}\right|_{z^{\prime}, P^{\prime}, \sigma}=0$. Moreover, $\widehat{H}$ is holomorphic in $\mathscr{P} u$.

Proof. The first equality follows directly from the definitions. For the second we calculate

$$
\begin{aligned}
\left.\partial_{\bar{u}} \widehat{H}\right|_{z^{\prime}, P^{\prime}, \sigma} & =\underbrace{\partial_{\bar{u}} /\left.\Lambda^{n+1, \ldots \mid}\right|_{0, P^{\prime}, \sigma} \cdot \overline{\mathscr{P}}}_{\stackrel{(3,2)}{=}=0}+\underbrace{\partial_{P^{\prime}} /\left.\mathbf{\Lambda}^{n+1, \ldots}\right|_{0, P^{\prime}, \sigma}}_{\left(0 \mid I_{d}\right)} \cdot \partial_{\bar{u}} /\left.\mathbf{\Lambda}^{*}\right|_{z^{\prime}, P^{\prime}, \sigma} \\
& =\partial_{\bar{u}} / \mathbf{\Lambda}^{* n+1, \ldots, m}=\left.\partial_{\bar{u}}\left(s^{*}+i \varphi\left(z^{\prime}+\left.\Lambda\right|_{\sigma} \mathscr{Q}\left(u-z^{\prime}\right), s^{*}\right)\right)\right|_{z^{\prime}, P^{\prime}, \sigma} \\
& =\left(1+i \varphi_{s}\left(z^{\prime}+\left.\Lambda\right|_{\sigma} \mathscr{Q}\left(u-z^{\prime}\right), s^{*}\right)\right) \partial_{\bar{u}} s^{*}+\left.i \phi_{\bar{u}}\left(u, z^{\prime}, s^{*}, \sigma\right)\right|_{z^{\prime}, P^{\prime}, \sigma} \\
& =0 \in M_{d, n}(\mathbf{C}), \quad \text { thanks to our choice of } s^{*} .
\end{aligned}
$$

For $1 \leq j, k \leq n$ we get

$$
\begin{aligned}
\left.\partial_{\bar{u}_{j} \bar{u}_{k}}^{2} \widehat{H}\right|_{z^{\prime}, P^{\prime}, \sigma} & =\left.\partial_{\bar{u}_{j}}\left(\left(1+i \varphi_{s}\right) \partial_{\bar{u}_{k}} s^{*}+i \phi_{\bar{u}_{k}}\right)\right|_{z^{\prime}, P^{\prime}, \sigma} \\
& =\left.\left(1+i \varphi_{s}\right) \partial_{\bar{u}_{j} \bar{u}_{k}}^{2} s^{*}\right|_{z^{\prime}, P^{\prime}, \sigma}+\mathscr{H}_{j k}\left(P^{\prime}, \sigma\right),
\end{aligned}
$$

where we chose the calligraphic letter $\mathscr{H}$ to denote a quite horrible tensor taking values in $\operatorname{Lin}^{2}\left(\mathbf{C}^{n}, \mathbf{C}^{d}\right)$. Fortunately, we do not need to calculate it. It suffices to observe that $\mathscr{H}$ is invariant by $\overline{\mathscr{Q}}$, that is, $\forall_{1 \leq l \leq d} \mathscr{H}^{l}={ }^{t} \overline{\mathscr{Q}} H^{l} \overline{\mathscr{Q}}$. Therefore we can choose, in (3.7),

$$
\partial_{\bar{u}_{j} \bar{u}_{k}}^{2} s^{*}=\left(\begin{array}{c}
\left.{ }^{t} \overline{\mathscr{Q}} H^{(1)} \overline{\mathscr{Q}}\right)_{j k} \\
\vdots \\
\left(\overline{\mathscr{Q}} H^{(d)} \overline{\mathscr{Q}}\right)_{j k}
\end{array}\right),
$$

equally monstrous to assure that the sum (3.9) disappears.

Remark 3.10 implies the existence of a smooth map $h: \widehat{\Omega} \rightarrow M_{d, n(}(\mathbf{C})$, holomorphic in $\mathscr{P} u$, such that

$$
\widehat{H}=h\left(u-z^{\prime}\right)+O\left(\left|\mathscr{Q}\left(u-z^{\prime}\right)\right|^{3}\right) .
$$

Similarly, Remark 3.8 allows us to find a smooth map $A: \widehat{\Omega} \rightarrow \mathrm{Gl}_{n}(\mathbf{C})$, holomorphic in $\mathscr{P} u$, with

$$
z-z^{\prime}=A\left(u-z^{\prime}\right)+O\left(\left|\mathscr{Q}\left(u-z^{\prime}\right)\right|^{3}\right) .
$$


We now put $i \widehat{\alpha}:=h \cdot A^{-1}: \widehat{\Omega} \rightarrow M_{d, n}(\mathbf{C})$ and obtain

$$
\widehat{H}\left(u, P^{\prime}, \sigma\right)=i \widehat{\alpha}\left(u, P^{\prime}, \sigma\right) \cdot\left(z\left(u, P^{\prime}, \sigma\right)-z^{\prime}\right)+O\left(\left|\mathscr{Q}(\sigma)\left(u-z^{\prime}\right)\right|^{3}\right) .
$$

Definition 3.11. We introduce the smooth maps

$$
\begin{aligned}
& H: \Omega \times \Sigma \times \mathscr{C} \rightarrow \mathbf{C}^{d} ; \quad\left(z, P^{\prime}, \sigma\right) \mapsto \widehat{H} \circ \mathscr{Z}^{-1}\left(z, P^{\prime}, \sigma\right), \\
& \alpha: \Omega \times \Sigma \times \mathscr{C} \rightarrow M_{d, n}(\mathbf{C}) ; \quad\left(z, P^{\prime}, \sigma\right) \mapsto \widehat{\alpha} \circ \mathscr{Z}^{-1}\left(z, P^{\prime}, \sigma\right) .
\end{aligned}
$$

With $\widehat{\alpha}, \alpha$ is holomorphic and positive homogeneous of degree zero in $\sigma$. The condition (3.6) follows now from the fact that $\widehat{\alpha}$ is holomorphic in $\mathscr{P} u$. Indeed, since

$$
\alpha=\left(\begin{array}{c}
\alpha^{1} \\
\vdots \\
\alpha^{d}
\end{array}\right)
$$

does not depend on $s$, we have for all $1 \leq l \leq d, 1 \leq i, j \leq n, L_{j} \alpha_{i}^{l}\left(P, P^{\prime}\right)=$ $\left.\partial_{\bar{z}_{j}} \alpha_{i}^{l}\right|_{P, P^{\prime}}$ and the holomorphy of the map $u \mapsto \widehat{\alpha}^{l}\left(\mathscr{P} u, P^{\prime}, \sigma\right)$ yields

$$
\begin{gathered}
0=\partial_{\bar{u}} \widehat{\alpha}^{l} \cdot \overline{\mathscr{P}}=\partial_{z} \alpha^{l} \underbrace{\partial_{\bar{u}} \overline{\mathscr{P}}}_{=0}+\partial_{\bar{z}} \alpha^{l} \underbrace{\partial_{\bar{u}} \bar{z}}_{\text {invertible }} \overline{\mathscr{P}} \\
\Rightarrow\left(L_{j}(\sigma \alpha)_{i}\right) \underbrace{\partial_{\bar{u}} \bar{z} \overline{\mathscr{P}}(\sigma)}_{\text {rank } q}=0 \\
\Rightarrow \operatorname{rank}\left(L_{j}(\sigma \alpha)_{i}\right) \leq n-q .
\end{gathered}
$$

The reader might wonder what happened to our main hypothesis (3.4). So far it has not been used. Let $z=z\left(u, P^{\prime}, \sigma\right)$. If we put $P^{\prime \prime}:=/ \Lambda^{*}\left(u, P^{\prime}, \sigma\right)$, then we can write $z=\Lambda\left(\mathscr{P}\left(u-z^{\prime}\right), P^{\prime \prime}, \sigma\right)$ and (3.4) yields the inequality

$$
\begin{aligned}
& 0<\sigma\left[\varphi\left(z, \operatorname{Re} / \Lambda^{n+1, \ldots, m}\right)-\operatorname{Im} / \Lambda^{n+1, \ldots}\left(\mathscr{P}\left(u-z^{\prime}\right), P^{\prime \prime}, \sigma\right)\right] \\
& \stackrel{\text { Def.3.9 }}{=} \sigma\left[\varphi\left(z, s^{\prime}+\operatorname{Re} H\right)-\operatorname{Im} H-\varphi\left(z^{\prime}, s^{\prime}\right)-\left|\mathscr{Q}\left(u-z^{\prime}\right)\right|^{2} \frac{{ }^{t} \sigma}{\langle\sigma\rangle}\right],
\end{aligned}
$$

where $H$ stands for $H\left(z, P^{\prime}, \sigma\right)$ and $\langle\sigma\rangle$ for $\sqrt{\sigma \cdot{ }^{t} \sigma}$. We conclude that for $\langle\sigma\rangle=1$ we have $\sigma\left[\varphi\left(z, s^{\prime}+\operatorname{Re} H\right)-\varphi\left(z^{\prime}, s^{\prime}\right)-\operatorname{Im} H\right] \geq\left|\mathscr{Q}\left(u-z^{\prime}\right)\right|^{2}$, with strict inequality unless $\mathscr{P}\left(u-z^{\prime}\right)=0$. But then of course we obtain

$$
\sigma\left[\varphi\left(z, s^{\prime}+\operatorname{Re} H\right)-\varphi\left(z^{\prime}, s^{\prime}\right)-\operatorname{Im} H\right]>\frac{1}{2}\left|\mathscr{Q}\left(u-z^{\prime}\right)\right|^{2},
$$

where now the inequality is strict unless $u=z^{\prime}$.

Definition 3.12. Let $M>0$ be a number greater than all second derivatives of $\varphi$ in $\mathscr{U}$. We then define a map $\mu: \mathscr{U} \times \mathscr{U} \times \mathscr{C} \rightarrow M_{d}(\mathbf{C})$ by letting $\mu\left(z, s, z^{\prime}, s^{\prime}, \sigma\right):=\mu\left(P, P^{\prime}, \sigma\right):=I_{d}-\left.i \varphi_{s}\right|_{z, s}-i M\left({ }^{t} \sigma /\langle\sigma\rangle\right)^{t}(s-\tilde{s})$, where $\tilde{s}:=s^{\prime}+\operatorname{Re}\left\{i \alpha\left(z, z^{\prime}, s^{\prime}, \sigma\right) \cdot\left(z-z^{\prime}\right)\right\}$.

For $\sigma \in \mathbf{R}_{d}$, the real part of $\mu$ is the identity matrix. It remains to show property (3.5). Let $\sigma$ be real, $|\sigma|=1$. In the following we simply write $\alpha, \mu$, and $H$ for $\alpha\left(z, z^{\prime}, s^{\prime}, \sigma\right), \mu\left(z, s, z^{\prime}, s^{\prime}, \sigma\right)$ and $H\left(z, P^{\prime}, \sigma\right)$, respectively and show that

$\begin{array}{ll}\text { (1) } \operatorname{Im}\left\{\sigma \mu\left[w-w^{\prime}-i \alpha\left(z-z^{\prime}\right)\right]\right\}= & \sigma \operatorname{Im} \mu(s-\tilde{s})+\sigma(\varphi(z, s)-\varphi(z, \tilde{s})) \\ & +\sigma\left(\varphi(z, \tilde{s})-\varphi\left(z, s^{\prime}+\operatorname{Re} H\right)\right) \\ & \\ \text { (3) } & +\sigma\left(\varphi\left(z, s^{\prime}+\operatorname{Re} H\right)-\operatorname{Im}\left\{w^{\prime}+H\right\}\right) \\ \text { (4) } & +\sigma \operatorname{Im} H-\sigma \operatorname{Im}\left\{i \alpha\left(z-z^{\prime}\right)\right\}\end{array}$


is strictly positive, except when $P=P^{\prime}$. The term $(1)$ is $>0$ by Taylor's theorem, and vanishes iff $s=\tilde{s}$. By (3.10), $H=i \alpha\left(z-z^{\prime}\right)+O\left(\left|\mathscr{Q}\left(u-z^{\prime}\right)\right|^{3}\right)$, hence the absolute value of (2) plus (4) is an $O\left(\left|\mathscr{Q}\left(u-z^{\prime}\right)\right|^{3}\right)$. On the other hand, the holoconvexity gave us $(3.11)$, i.e., the term (3) is $>\frac{1}{2}\left|\mathscr{Q}\left(u-z^{\prime}\right)\right|^{2}$. Thus we see that (2) + (3) + (4) $>0$, and zero iff $z=z^{\prime}$. The sum of all four terms is positive and vanishes only when $s=\tilde{s}$ and $z=z^{\prime}$, that is precisely when $P=P^{\prime}$.

This completes the proof of Lemma 3.6.

The last step in the proof of Theorem 3.4 is almost identical to the end of the proof of Theorem 2.7. We apply the homotopy formula to our $C^{1}$-form $f$ for the map

$$
a: \mathscr{U} \times \mathscr{U} \times \mathscr{C} \rightarrow \mathbf{C}_{n} ; \quad\left(z, s, z^{\prime}, s^{\prime}, \sigma\right) \mapsto \sigma \cdot \alpha\left(z, z^{\prime}, s^{\prime}, \sigma\right) .
$$

Thanks to Theorems 1.4 and 1.5 we obtain the equation

$$
\begin{aligned}
\mathscr{I}^{\varphi} f= & \bar{\partial}_{\Sigma}\left((-1)^{n-q+1} \mathscr{K}_{\mathscr{Q}}^{a, \varphi} f\right)+(-1)^{n-q} \mathscr{K}_{\mathscr{O}}^{a, \varphi}\left(\bar{\partial}_{\Sigma} f\right) \\
& +(-1)^{n-q+1} \mathscr{K}_{\partial \mathscr{\mathscr { Q }}}^{a, \varphi} f .
\end{aligned}
$$

Again adopting the shorthand $\int_{0}^{1} \varphi_{s}$ instead of $\int_{0}^{1} \varphi_{s}\left(z, \tau s+(1-\tau) s^{\prime}\right) d \tau$, which now is a $d \times d$ matrix, we define the pseudodifferential operator $\mathbf{I}^{\varphi}$ by

$$
\mathbf{I}^{\varphi} f_{J}(z, s):=\int_{\mathscr{T}} \int_{\mathbf{R}_{d}} e^{i \sigma\left(s-s^{\prime}\right)} f_{J}\left(z, s^{\prime}\right) \frac{\operatorname{det}\left(1+i \varphi_{s}\left(z, s^{\prime}\right)\right)}{\operatorname{det}\left(1+i \int \varphi_{s}\right)} \frac{d \sigma}{(2 \pi)^{d}} d s^{\prime}
$$

and, of course,

$$
\mathbf{I}^{\varphi} f(z, s):=\sum_{|J|=n-q+1} \mathbf{I}^{\varphi} f_{J}(z, s) d \bar{z}_{J} \wedge d z \wedge d w .
$$

Lemma 3.13. As $\varepsilon \downarrow 0$, the integral

$$
\int_{\sigma \in \mathbf{R}_{d}} e^{-\varepsilon\langle\sigma\rangle^{2}} \mathscr{J}^{\varphi} f(z, s, \sigma) \frac{d \sigma}{(2 \pi)^{d}}
$$

converges to $\mathbf{I}^{\varphi} f(z, s)$, and the operator $\mathbf{I}^{\varphi}$ is congruent to the identity modulo regularizing operators. Moreover, the limit

$$
\begin{aligned}
& \bullet \mathbf{K}_{\mathscr{O}}^{a, \varphi} f(z, s):=\lim _{\varepsilon \downarrow 0} \int_{\sigma \in \mathbf{R}_{d}} e^{-\varepsilon\langle\sigma\rangle^{2}} \mathscr{K}_{\mathscr{\theta}}^{a, \varphi} f(z, s, \sigma) d \sigma /(2 \pi)^{d} \\
& \text { exists in } C^{0}\left(\Sigma, \Lambda^{m, n-q}\right) . \\
& \bullet \mathbf{K}_{\mathscr{O}}^{a, \varphi} \bar{\partial}_{\Sigma} f(z, s):=\lim _{\varepsilon \downarrow 0} \int_{\sigma \in \mathbf{R}_{d}} e^{-\varepsilon\langle\sigma\rangle^{2}} \mathscr{K}_{\mathscr{\theta}}^{a, \varphi} \bar{\partial}_{\Sigma} f(z, s, \sigma) d \sigma /(2 \pi)^{d} \\
& \text { exists in } C^{\infty}\left(\Sigma, \Lambda^{m, n-q+1}\right) . \mathbf{K}_{\partial \mathscr{\theta}}^{a, \varphi} f(z, s):=\lim _{\varepsilon \downarrow 0} \int_{\sigma \in \mathbf{R}_{d}} e^{-\varepsilon\langle\sigma\rangle^{2}} \mathscr{K}_{\partial \mathscr{\theta}}^{a, \varphi} f(z, s, \sigma) d \sigma /(2 \pi)^{d} \\
& \text { exists in } C^{\infty}\left(\Sigma, \Lambda^{m, n-q+1}\right) .
\end{aligned}
$$

The proof of this lemma can be found in [P]. It is almost identical to the proof of Lemma 2.14. However, here we integrate over the whole space $\mathbf{R}^{d}$ and not only over conic subsets, because otherwise in the process of changing the contour from $\{\sigma\}$ to $\left\{\sigma \cdot\left(1+i \int \varphi_{s}\right)^{-1}\right\}$ or $\{\sigma \mu\}$ respectively, we would 
obtain contributions from the boundaries about which our crucial Lemma 3.6 does not give any information.

Conclusion. The homotopy formula allows us to write (cf. (3.12))

$$
\begin{aligned}
\mathbf{I}^{\varphi} f(z, s)= & \bar{\partial}_{\Sigma}\left((-1)^{n-q+1} \mathbf{K}_{\mathscr{O}}^{a, \varphi} f\right)+(-1)^{n-q} \mathbf{K}_{\mathscr{O}}^{a, \varphi} \bar{\partial}_{\Sigma} f \\
& +(-1)^{n-q+1} \mathbf{K}_{\partial \mathscr{\theta}}^{a, \varphi} f .
\end{aligned}
$$

Thanks to our choice of the map $a$ and using a change of the domain of integration induced by $\mu$ we obtained Lemma 3.13 stating that the currents $\mathbf{K}_{\mathscr{Q}} \bar{\partial}_{\Sigma} f$ and $\mathbf{K}_{\partial \mathscr{O}} f$ are smooth. Since $\mathbf{I}^{\varphi} f$ is congruent to the identity (modulo regularizing operators) we have $\operatorname{WF}\left(\mathbf{I}^{\varphi} f-f\right)=\varnothing$. Letting $u:=(-1)^{n-q+1} \mathbf{K}_{\mathscr{O}}^{a, \varphi} f$ we have therefore shown that

$$
\begin{aligned}
& \mathrm{WF}\left(f-\bar{\partial}_{\Sigma} u\right)=\mathrm{WF}\left(\mathbf{I}^{\varphi} f-\bar{\partial}_{\Sigma} u\right)=\varnothing \\
& \quad \Leftrightarrow f \text { is cohomologous to a smooth form. }
\end{aligned}
$$

Thus we have proven that on a $q$-holoconvex manifold $\Sigma$ of arbitrary codimension the operator $\bar{\partial}_{\Sigma}$ is $(n-q+1)$-hypoelliptic. In order to get the conclusion that $\bar{\partial}_{\Sigma}$ is $(q-1)$-hypoelliptic we need to replace $L_{j}$ by $L_{j}^{\prime}$ in inequality (3.6) so that in (3.12) no error term will show up. This can be done by interchanging the roles played by $(z, s)$ and $\left(z^{\prime}, s^{\prime}\right)$ in the proof of Lemma 3.6. Of course we do not want to invert the direction of inequality (3.5) because that would spoil completely the convergence needed in Lemma 3.13. But using $/ \Lambda\left(u, P^{\prime},-\sigma\right)$ instead of $/ \Lambda$ in Definition 3.9 we can invert the crucial inequality (3.11) so that then (3.5) remains unchanged.

Finally, observing that the hypothesis of $q$-holoconvexity gets stronger as $q$ increases, we see that indeed we have shown the $q^{\prime}$-hypoellipticity and $\left(n-q^{\prime}\right)$ hypoellipticity for all integers $q^{\prime}<q$.

\section{REFERENCES}

[AHe] R. A. Airapetyan and G. M. Henkin, Integral representations of differential forms on CauchyRiemannn manifolds and the theory of CR-functions, Uspekhi Mat. Nauk 39:3 (1984), 39106; English transl., Russian Math. Surveys 39:3 (1984), 41-118.

[AH] A. Andreotti and C. D. Hill, E. E. Levi convexity and the Hans Lewy problem, Ann. Scuola Norm. Sup. Pisa 26 (1972), 325-363 and 747-806.

[FS] G. B. Folland and E. M. Stein, Estimates for the $\bar{\partial}_{b}$ complex and analysis on the Heisenberg group, Comm. Pure Appl. Math. 27 (1974), 429-522.

[He] G. M. Henkin, The Lewy equation and analysis on pseudoconvex manifolds, Russian Math. Surveys 32:3 (1977), 59-13.

[H1] L. Hörmander, Pseudodifferential operators and non-elliptic boundary problems, Ann. of Math. (2) 83 (1966), 129-209.

[H2] _ The analysis of linear partial differential operators. I, Springer, 1983.

[KN] J. J. Kohn and L. Nirenberg, A pseudoconvex domain not admitting a holomorphic support function, Math. Ann. 201 (1973), 265-268.

[M] H.-M. Maire, Necessary and sufficient conditions for maximal hypoellipticity of $\bar{\partial}_{b}$, Lecture Notes in Math., vol. 1324, Springer, 1988, pp. 178-185.

[NR] A. Nagel and J.-P. Rosay, Approximate local solutions of $\bar{\partial}_{b}$, but nonexistence of homotopy formula, for (0, 1)-forms on hypersurfaces in $\mathbf{C}^{n}$, Duke Math. J. 58 (1989), 823-827.

[P] J. A. Petersen, On the hypoellipticity of the tangential Cauchy-Riemann operator, Thesis, Rutgers Univ., May 1990. 
[T1] François Trèves, Pseudodifferential and Fourier integral operators, Plenum Press, 1980.

[T2] _- Hypoanalytic structures, Contemp. Math. 27 (1984), 23-44.

[T3] _ Homotopy formulas in the tangential Cauchy-Riemann complex, Mem. Amer. Math. Soc., vol. 87 (1990), no. 434.

Am Sachsenberg 5, Wentorf bei Hamburg, Germany 